\title{
La breve vita dello psykter. Parabola dell'habrosyne sullo scorcio dell'arcaismo*
}

\section{Catia Trombetti}

Miracolo della tecnica, vaso galleggiante dalla forma e funzione peculiari, lo psykter ${ }^{1}$ appare improvvisamente nelle officine dei ceramisti attici attorno al 530 a.C., per scomparire nel giro di due generazioni. Gli esemplari che conosciamo superano di poco il centinaio, sono quasi tutti di pregio e vantano, non a caso, le firme dei ceramisti e dei pittori più famosi del tempo ${ }^{2}$.

Negli anni Settanta del secolo appena trascorso S. Drogou mette a punto uno studio dettagliato su questa forma ceramica, basato sul riesame di tutta la documentazione disponibile. La studiosa calca per prima il terreno di una nuova interpretazione del vaso, dimostrando in via definitiva come lo psykter fosse destinato a contenere neve o acqua ghiacciata per freddare il vino nel cratere durante il simposio. Il suo contributo permette di rovesciare la tradizionale e consolidata prospettiva, per cui lo psykter sarebbe stato destinato a contenere il vino e il cratere la sostanza refrigerante ${ }^{3}$. Stupisce, tuttavia, come ancora oggi parte della comunità scientifica rimanga ancorata ai vecchi schemi ${ }^{4}$, nonostante tutte le prove portate a loro supporto siano passibili di critica.

Innanzitutto, le controverse immagini sulla ceramica attica, che ritraggono personaggi nell'atto di attingere direttamente dallo psykter in contesto simposiaco, non costituiscono alcuna testimonianza a favore della possibilità che il vaso

\footnotetext{
* Questo contributo trae origine da un intervento effettuato in occasione del Seminario di Studi “Oggetti: forme funzioni, interpretazioni”, Facoltà di Lettere e Filosofia - Sala delle Adunanze, palazzo Manzoni, Università degli Studi di Perugia, 14-16 dicembre 2010. Ringrazio il Prof. Gian Luca Grassigli per l'invito al convegno, il Prof. Massimo Nafissi per aver letto con attenzione il manoscritto, fornendomi preziose indicazioni bibliografiche, e il Dott. Marco Giuman per le consulenze sul miele.

${ }^{1}$ Il termine "psykter" indica nelle fonti un recipiente refrigeratore, ma non sembra mai designare la forma ceramica oggetto di questo studio. Con riferimento ad essa, entra per la prima volta e definitivamente nella letteratura archeologica con la monografia di W. KLEIN “Euphronios” del 1886. Cfr. ThesGrL s.v. psykter; DROUGOU 1975, pp. 7, 28-29; VIERNEISEL 1990, p. 259. La capacità del vaso di galleggiare è stata verificata sperimentalmente attraverso l'impiego di prototipi. A tale proposito si veda: DROUGOU 1975, p. 108 nota 31.

2 Il presente contributo si basa sul riesame di tutto il materiale edito, a partire dal catalogo degli psykteres realizzato da S. Drougou nel 1975, integrato ed aggiornato con i dati disponibili nel Beazley Archive on-line. Con le stesse modalità, sono state analizzate anche le rappresentazioni degli psykteres che compaiono su diverse forme ceramiche. Cfr. tavole 1-2: dalla prima sono stati esclusi 26 esemplari a vernice nera (DROUGOU 1975, pp. 21-25 a1-a2, b1-b24), 10 frammenti non classificabili (DROUGOU 1975, pp. 25-26 c1-c10) e l'unico esemplare in bronzo sinora attestato (BoTHMER 1961, pp. 141-144; DrougOU 1975, pp. 26-27). È significativo che i pezzi a vernice nera attualmente noti costituiscano meno di un quarto dell'intera produzione.

${ }^{3}$ DrougOu 1975, pp. 8-9, 31-33.

4 BOARDMAN 1977, p. 306, recensendo la monografia della Drougou, si dichiara concorde con l'ipotesi della studiosa, respinta invece da MOORE - PHILIPPIDES 1986, pp. 20-22; VIERNEISEL 1990, pp. 259-264 e SCHREIBER 1999, pp. 219-221. In BOARDMAN 2004, tuttavia, la fig. 276 a p. 250 viene così descritta "Un ragazzo attinge il vino con un mestolo da uno psykter posto all'interno di un cratere a calice”. Si tratta dell'oinochoe a figure nere di Kleisophos, di cui parleremo più avanti (pp. 19-20). Il vaso è curiosamente chiamato in causa anche in DROUGOU 1975, p. 32 a supporto della tesi opposta, dal momento che in questo caso il mestolo si trova in modo piuttosto evidente fra la spalla dello psykter e l'orlo del cratere, non sopra lo psykter. Sostanzialmente, pertanto, il giovane inserviente sta attingendo dal cratere a calice, non dallo psykter! Incerta anche la posizione di I. SCHEIBLER, che accoglie l'idea della Drougou (SCHEIBLER 1995, p. 23), per poi respingerla (SCHEIBLER 2001, p. 523).
} 
contenesse vino. I comasti o gli inservienti potevano, infatti, attingere anche neve intatta o sciolta - da aggiungere al vino già versato nelle coppe per rinfrescarlo ${ }^{5}$. A tale proposito, come vedremo, sembra dirimente un epigramma di Simonide che avremo modo di commentare dettagliatamente ${ }^{6}$. Una lekythos del Pittore di Pan, inoltre, mostra un'ancella con una patera, intenta ad attingere con un mestolo dal grande skyphos che le porge la padrona di casa seduta di fronte a lei. A terra, fra le due donne, si erge uno psykter chiuso da un coperchio ${ }^{7}$. La scena, di difficile esegesi, è stata letta come una libagione in ambiente domestico ${ }^{8}$. Ad ogni modo, poichè il vino necessario all'operazione viene attinto dallo skyphos, lo psykter, ben tappato, deve per forza contenere dell'altro e alludere ad un momento precedente o successivo a quello rappresentato nel "fotogramma.

Risolutive a favore della Drougou sono, d'altra parte, alcune considerazioni relative alle peculiarità morfologiche del vaso e al suo rapporto privilegiato con il cratere a calice ${ }^{9}$. Lo psykter, infatti, è un vaso dal collo stretto, dotato di coperchio, che difficilmente raggiunge notevoli dimensioni ${ }^{10}$. Si tratta pertanto di un recipiente ideale a contenere neve e mantenerne a lungo le proprietà ${ }^{11}$, ma inadatto ad accogliere le quantità di vino necessarie ad un agevole svolgimento della prassi simposiaca ${ }^{12}$. Se il vino riempisse lo psykter e la neve il cratere, il primo sarebbe costantemente svuotato, mentre il secondo vedrebbe la neve sciogliersi troppo rapidamente. Gli inservienti avrebbero dovuto ininterrottamente rimboccare l'uno e l'altro. Non è immaginabile, peraltro, che nel corso di un incontro conviviale vi fosse una tale disponibilità di neve da riempire crateri su crateri. Dovremmo pensare, come è stato

${ }^{5}$ Per la documentazione iconografica si veda la tavola 2. Nei casi in cui la presenza dello psykter si colloca al di fuori dello spazio simposiaco è facile pensare a fasi preliminari, come è chiaro ad esempio nel n. 16. Le scene in cui il vaso appare in assenza del cratere, invece, sono da considerare come pars pro toto, rappresentazioni sintetiche e allusive ad un contesto concettualmente più ampio. In nessun caso, infatti, abbiamo scene simposiache vere e proprie, dove alla coppia funzionale cratere/psykter si sostituisce il solo psykter (così MOORE - PHILIPPIDEs 1986, p. 21 nota 4). Generalmente si tratta di scene di komos (nn. 8, 11, 13, 14, 17, 19, 22, 23), un momento successivo a quello del simposio, nel cui contesto sono previsti anche atteggiamenti decisamente poco convenzionali. In una simile circostanza, è comprensibile che lo psykter potesse essere estratto dal cratere, recato in mano nella danza, trasportato, sollevato, utilizzato in modo del tutto decontestualizzato rispetto alla prassi simposiaca. Va sottolineato, infine, come anche la presunta ed eventuale difficoltà ad attingere vino dal cratere per la presenza dello psykter (così VIERNEISEl 1990, p. 263) sia un argomento debole a favore della teoria tradizionale, poiché lo stesso discorso potrebbe valere anche per l'acqua.

${ }^{6}$ Si veda la p. 21 di questo contributo.

${ }^{7}$ Cfr. tav. 2 n. 21.

${ }^{8}$ GREIFENHAGEN 1961, p. 125.

${ }^{9}$ Drougou 1975, pp. 32-33.

${ }^{10}$ L'altezza del vaso varia tra i 13 e i $40 \mathrm{~cm}$ ca., il diametro della bocca tra gli 8 e i $18 \mathrm{~cm}$ ca. Cfr. DrougOu 1975, pp. 12-25.

${ }^{11}$ É difficile immaginare che la funzione del coperchio sia stata quella di proteggere il vino dalla caduta di corpi estranei ed evitarne la fuoriuscita durante il trasporto, come vorrebbe VIERNEISEL 1990, p. 263. Una simile preoccupazione igienica, infatti, avrebbe dovuto, precedentemente e successivamente all'impiego dello psykter, a maggior ragione riguardare anche i crateri (dal diametro decisamente più ampio), eppure non sembra esservi alcun tipo di documentazione a proposito. Inoltre, nel corso del simposio, se pure il vino nello psykter fosse stato protetto, corpi estranei avrebbero potuto in ogni caso cadere nel cratere, che conteneva comunque liquido da mescere e destinato ad essere ingerito. In quanto al problema del trasporto, lo studioso si contraddice, sostenendo contestualmente e giustamente che la quasi generale assenza di anse negli psykteres o la presenza al massimo di piccole orecchie o tubuli testimonierebbe come i contenitori non fossero destinati al trasporto di neve (cosa che in effetti nessuno aveva mai supposto), ma a galleggiare all’interno di altri contenitori.

${ }^{12}$ Nonostante il rapporto di 3:1 in cui sono generalmente mescolati acqua e vino. Sul dato porta l'attenzione VIERNEISEL 1990, p. 261. 
suggerito ${ }^{13}$, che in realtà al posto della neve si utilizzasse fondamentalmente acqua fresca per raffreddare, ma come già ricordato sono le fonti ad indicare esplicitamente anche l'impiego di neve nei simposi. Va tenuto presente, infine, che il cratere è il vaso in cui l'acqua ed il vino sono mescolati nella misura stabilita dal simposiarca, quello da cui si attinge per la libagione agli dei e la distribuzione ai commensali ${ }^{14}$. Anche dal punto di vista ideologico, quindi, considerato il valore simbolico dell'oggetto nella società greca quale sintesi dei valori legati alla pratica conviviale, appare estremamente difficile immaginare il ruolo del vaso declassato a contenitore d'acqua, con lo scomodo inconveniente di dover ripetutamente mescolare acqua e vino nelle coppe per ciascun commensale. Per quale ragione rendere così poco agevole l'operazione della mescita?

Lo psykter deve essere pensato come un valore aggiunto, non come qualcosa in grado di scardinare schemi tradizionali, prontamente ripristinati in occasione della sua scomparsa. Se così non fosse, per il cratere a calice, una forma che nasce ma non muore con lo psykter, dovremmo addirittura ipotizzare un cambio di funzione in occasione della scomparsa di quest'ultimo.

La posizione della Drougou, perciò, si configura ad oggi come la più logica ed economica dal punto di vista esegetico. La breve monografia della studiosa, tuttora valida anche per quanto concerne l'evoluzione morfologica dello psykter, sembra in realtà discutibile soltanto per alcune considerazioni relative alla scomparsa del vaso dal mercato dopo il 470 a.C.

Sostenere che lo psykter divenga improvvisamente fuori moda in qualità di oggetto accessorio e facilmente sostituibile ${ }^{15}$, infatti, significa non tener conto dei risvolti sociali che generano o viceversa ostacolano l'affermarsi di certe tendenze ${ }^{16}$, applicando al mondo antico categorie forgiate su misura per la realtà contemporanea $^{17}$. Se sono le pratiche sociali, attraverso l'impiego di segni codificabili, a disegnare i contorni dell'appartenenza e a conferire struttura alla collettività $^{18}$, diviene allora impossibile immaginare una fruizione nella distrazione di oggetti significativi come gli psykteres, in un contesto, come vedremo, socialmente molto ben definito.

Un'esegesi come quella proposta dalla studiosa e accettata dalla letteratura scientifica si scontra, oltretutto, con la continuità di produzione di altre forme ceramiche, che pure vedono la luce con lo psykter, come lo stamnos o il cratere a calice $^{19}$.

L'episodio dello psykter diviene comprensibile, in realtà, solo facendo luce sugli aspetti che permettono di circoscriverne la fruizione e definirne la semantica nella diacronia. Innanzitutto, sarà da riflettere su un dato qualitativo: la produzione di psykteres è pressoché esclusiva delle officine dei Pionieri e dei loro seguaci, artigiani

\footnotetext{
${ }^{13}$ VIERNEISEL 1990, p. 262.

${ }^{14}$ Cfr. Lissarrague 1990, pp. 196-207.

${ }^{15}$ Drougou 1975, pp. 8, 34. L’opinione è accolta in VIERNEISEL 1990, pp. 259-260.

${ }^{16}$ La moda è comunicazione, un linguaggio dell'effimero che affonda inevitabilmente le radici nel proprio milieu culturale. Nell'ambito degli ormai numerosi studi sul tema, si vedano: SEGRE REINACH 2005, pp. 3-32, con bibliografia; LA CECLA 2007, pp. 5-8.

${ }^{17}$ Cfr. GRASSIGLi 1999, pp. 447-456, in particolare la nota 24.

${ }^{18}$ DuplOuY 2006, p. 255.

${ }^{19}$ Sulle due forme ceramiche si vedano: PHILIPPAKI 1967; SISTO 2006, pp. 151-163; SHAPIRO 1985, pp. 261-264; FrANK 1990; Huber 1992, pp. 57-72; RoBERTSON 1996, p. 26. Per la stessa ragione non regge il paragone che la DROUGOU, sottolineando i risvolti ludici cui lo psykter poteva dar luogo in contesto simposiaco, stabilisce fra l'invenzione del vaso e quella del kottabos, introdotto ad Atene nella seconda metà del VI sec. a.C. Cfr. Drougou 1975, p. 34.
} 
di fama e dal notevole peso socio-economico. In un secondo momento, dovremo valutare come gli anni dal 530 al 470 a.C. siano gli stessi che vedono anche la diffusione e la scomparsa dei cosiddetti “Anacreontic vases", nonché il progressivo scemare dei temi omoerotici nelle scene vascolari. Data la contemporaneità dei fenomeni in questione, infatti, si affaccia all'orizzonte la possibilità che possa esservi una relazione fra loro.

\section{Produzione e fruizione: un rapporto da decifrare}

I temi iconografici che si dipanano sugli psykteres sono quelli tipici della produzione vascolare contemporanea di più alto livello, sia a figure nere che rosse. Ad essere in scena è l'intero mondo del simposio, nella sua accezione dionisiaca e paideutica $^{20}$. In ottemperanza a quanto testimoniato anche dalla lirica arcaica, sullo scorcio del VI sec. a.C. i grandi temi mitologici vengono lentamente sostituiti dalla rappresentazione dei valori comunemente condivisi ${ }^{21}$. Le divinità e gli eroi lasciano spazio alla schiera degli uomini, sebbene proiettata nella dimensione paradigmatica e ideale prevista dai parametri dell'entourage aristocratico ${ }^{22}$.

Piuttosto che le iconografie, pertanto, a colpire sono la qualità dei soggetti rappresentati, la frequente volontà da parte del ceramista o del pittore di rendere unico il pezzo, apponendo firme ed iscrizioni volte a contestualizzare la scena ${ }^{23}$. Gli antroponimi che compaiono accanto ai personaggi raffigurati sono spesso rintracciabili nelle fonti letterarie o epigrafiche e facilmente riconducibili ai membri della jeunesse dorée ateniese di quegli anni ${ }^{24}$.

Un simile procedimento proietta l'immaginario relativo agli psykteres in una dimensione di classe dall'estremo valore simbolico, con richiami espliciti a quel mondo cui gli oggetti sono destinati. L'ampio numero di esemplari rinvenuti nei contesti sepolcrali etruschi ${ }^{25}$, del resto, ribadisce la circolazione di questi manufatti ad altissimo livello. Lo psykter è un vaso ricercato e originale, ha tutte le carte per entrare

\footnotetext{
${ }^{20}$ Tre sono i grandi temi iconografici: 1. il simposio/komos con 16 esemplari (tav. 1 nn. 14, 17-18, 21, 24-25, 28, 31-32, 43, 52, 55-56, 66-68, 71), cui sono riconducibili anche i soggetti più propriamente dionisiaci con12 esemplari (tav. $1 \mathrm{nn}$. 6-7, 12, 32, 34-36, 39, 51, 53, 58, 72); 2. la paideia, con 22 esemplari (tav. 1 nn. 8, 16, 19, 22-23, 26-27, 30, 33, 37, 40, 47-49, 59-65, 69); 3. la guerra con 9 esemplari (tav. 1 nn. 5, 9-11, 29, 31, 43, 50, 68).

${ }^{21}$ I nuovi motivi della poesia conviviale sono la parenetica, la politica e l'eros. Cfr. VETTA 1983, pp. xiii-lx, 53-54; KANTZIOS 2005, pp. 227-245. Così recita Anacr. fr. 33: “Non amo chi bevendo presso un cratere colmo / narra tumulti e risse e lacrimose guerre / ma chi mescendo delle Muse e d'Afrodite / gli splendidi doni, canta l'amabile gioia”, trad. GENTILI 1983, p. 89. Negli psykteres i temi mitologici si possono circoscrivere a 12 esemplari (tav. 1, nn. 4, 13, 15, 27, 38, 41-42, 44-45, 47, 57 e, forse, anche 70).

${ }^{22}$ Questa, la corretta prospettiva attraverso la quale leggere la maggioranza delle scene identificabili negli psykteres. La definizione di “Szene des Alltags” (così DrougOU 1975, pp. 100-101) appare ormai obsoleta, soprattutto per gli esemplari con riferimenti a personaggi o contesti reali.

${ }^{23}$ Cfr. tav. 1, nn. 15-16, 22-29, 33, 38-39. Di frequente, peraltro, alcuni pittori appongono sul vaso pseudo-iscrizioni, ad imitazione dei pezzi più famosi. Cfr. tav. 1, nn. 8, 17, 35-36, 52.

${ }^{24}$ WeBSTER 1972, p. 23; SHAPIRO 1980, pp. 289-293; ID. 1982, pp. 69-73; ID. 1983, pp. 305-310. Rimane suggestiva l'ipotesi di vedere in alcuni di questi vasi dei doni commissionati in occasione di incontri conviviali. GERNET 1968, pp. 151-173, 285-286 individua nel sistema ellenico, estremamente competitivo e basato sulle liturgie, il relitto di una struttura sociale fondata sullo scambio di doni. Da più parti, inoltre, è stato rilevato come negli ambienti di alto livello i retaggi di un simile sistema di relazioni possano persistere più fortemente. Cfr. GILL ET ALII 1998; NEER 2002, pp. 129-132; MAUSS $2002^{3}$, pp. 98-99.

${ }^{25}$ In particolare, Vulci e Spina. Cfr. Drougou 1975, p. 7 e nota 3.
} 
a pieno titolo nella categoria di quei beni appositamente selezionati dalle élites ai fini dell'autorappresentazione ${ }^{26}$.

Tali premesse non devono, tuttavia, illudere circa la possibilità di stabilire una corrispondenza biunivoca fra oggetto e fruitore in termini di classe sociale, proprio perché dobbiamo partire dall'assunto che ad uno stadio evoluto sono le pratiche sociali a definire l'appartenenza e non viceversa ${ }^{27}$.

La funzione strutturante di segni e modelli comportamentali nell'interazione sociale, del resto, è ampiamente dimostrata da un fenomeno estremamente dibattuto, che significativamente coinvolge anche alcuni psykteres. Si tratta di un procedimento adottato dai Pionieri su una serie di vasi di pregio, dove i medesimi ceramisti e pittori si autorappresentano in qualità di simposiasti, musicanti, atleti ed erastai, talora in compagnia di efebi d'alto rango ${ }^{28}$. Su uno psykter attribuito a Smikros, compare, ad esempio, il nome del ceramista Euphronios ${ }^{29}$. Cinque coppie di giovani, eromenos ed erastes, si susseguono nel campo visivo: Hegerthos e Andriskos, Ambrosios ed Euthydikos, Melas ed Antias, Euphronios e Leagros (acclamato kalos), seguiti da un'ultima coppia priva di nomi, ma caratterizzata da un atteggiamento erotico più palese rispetto alle altre [fig. 1].

La scena costituisce un vero e proprio sunto della paideia, col richiamo alla musica, alla palestra e all'eros omoerotico. Immediatamente perspicuo è anche l'espediente compositivo che utilizza personaggi anonimi e quindi prototipici per esplicitare quanto nelle altre coppie è semplicemente alluso. Meno immediato appare, invece, il significato della presenza di un ceramista nella cerchia di giovani aristocratici $^{30}$, per di più ritratto in un atteggiamento che in teoria doveva essergli precluso per motivi di classe ${ }^{31}$. Si è pertanto letta la scena come un omaggio di Smikros al ricchissimo ceramista Euphronios. Il Pittore avrebbe alluso in essa alle aspirazioni del proprio datore di lavoro ad uno stile di vita aristocratico e alla sua passione amorosa per Leagros ${ }^{32}$.

Simili rappresentazioni, in verità, piuttosto che parodie o tentativi dei banausoi di rivendicare l'accesso ad uno stile di vita elevato, in virtù del livello economico raggiunto $^{33}$, sembrano descrizioni paradigmatiche del reale. Alla luce di alcune

${ }^{26}$ In generale, sulle modalità di riconoscimento sociale: DUPLOUY 2006, pp. 28-35.

${ }^{27}$ DuPLOUY 2006, pp. 251-255.

28 “Ritratti”: $A R V^{2}$ 20.1, Para 322, Add ${ }^{2}$ 154; $A R V^{2} 1619.3$ bis, Para 322, Add² 152; ARV 63.90; NEER 2002, p. 133 P4 (tav. 1, n. 23); $A R V^{2}$ 23.7,1608, 1620, Para 323, Add² 155; ARV 23.1, Para 323, Add ${ }^{2}$ 154; $A R V^{2}$ 28.10, $A d d^{2}$ 156; $A R V^{2}$ 33.3. Iscrizioni/acclamazioni: $A B V$ 671; $A R V^{2}$ 32.1; $A R V^{2}$ 113.3; $A R V^{2}$ 23.7, 1608, 1620, Para 323, Add 155 ; $A R V^{2}$ 33.8, $A d d^{2}$ 214; $A R V^{2}$ 34.9; $A R V^{2}$ 27.3, Add ${ }^{2}$ 156; $A R V^{2}$ 18.1, $A d d^{2}$ 153; $A R V^{2}$ 26.1, Para 323, $A d d^{2}$ 155. Commento e descrizione in NeER 2002, pp. 87134.

${ }^{29}$ Getty Museum 82.AE.53; tav. 1, n. 23.

30 Tutti realmente vissuti e contemporanei di Euphronios. J. Frel ne rintraccia la testimonianza in diverse fonti letterarie ed epigrafiche. Cfr. MoON 1983, pp. 147-148.

${ }^{31}$ Un simile rapporto, che vedrebbe coinvolti in una relazione omoerotica un erastes dagli oscuri natali e un eromenos d'estrazione sociale elevata, avrebbe gettato disonore su quest'ultimo. A tale proposito si vedano: Dover 1989; WINKLER 1990, pp. 45-70; COHEN 1995, pp. 149-151. Numerose fonti stigmatizzano socializzazione e mescolanza fra diverse classi sociali, ribadendo l'inferiorità di coloro che praticano attività manuali e l'opportunità che ciascuno rispetti il proprio ruolo senza valicarne $\mathrm{i}$ confini. Cfr. Hdt. II 167; Xen. Oecon. IV 2-3; Plat. R. IV 420 d5-421 a2; Plat. Euth. 301 c-d.

${ }^{32}$ Così NeER 2002, pp. 100-102.

${ }^{33}$ L'acceso dibattito scientifico proietta concordemente i vasi in questione in una dimensione del tutto priva di agganci con la realtà. ROBERTSON 1996, p. 26 considera le scene un gioco dei pittori; per la STEINER 2007, pp. 258-262 si tratterebbe addirittura di parodie. Diversa la posizione di NEER 2002, pp. 
considerazioni, l'idea che alcuni parvenus potessero fregiarsi di compagnie di rango e di ciò vantarsi attraverso la propria arte, infatti, appare tutt'altro che peregrina ${ }^{34}$. In primo luogo, i nomi degli artigiani che compaiono sui vasi sono i medesimi che ritroviamo tra le iscrizioni dedicatorie dell'Acropoli ${ }^{35}$, un dato che autorizza ad immaginare, se pure per pochissimi individui, l'effettivo accesso ad uno stile di vita ispirato a modelli elitari. L'invettiva delle fonti di parte aristocratica contro i ceti emergenti, del resto, non fa che corroborare lo scenario di una progressiva e irreversibile frattura nel regno del privilegio ${ }^{36}$. L'obiezione circa la possibilità che un artigiano avesse l'opportunità di intrattenersi assieme ai membri dell'alta aristocrazia $^{37}$ cade, infine, di fronte al fatto che il fenomeno appare ristretto ad una cerchia di personaggi di fama. Alcuni ceramisti sembrerebbero anche simpatizzare o esibire reiteratamente, attraverso i propri manufatti, legami con i membri delle più importanti famiglie ateniesi ${ }^{38}$.

Il nome di Leagros, affiancato dall'acclamazione “kalos”, d'altra parte, frequente nei vasi di Euphronios e altri pittori della stessa cerchia ${ }^{39}$, , suggerisce la concretezza dell'amore per un ragazzo realmente esistito. A tale proposito vale la pena ricordare anche lo psykter dipinto e firmato da Euphronios, dove una delle quattro etere - Smikra, Palaisto, Sekline ed Agape - gioca al kottabos, utilizzandolo come Liebesorakel in onore di Leagros ${ }^{40}$ [fig. 2]. Se le scene dedicate al giovane siano poi da leggere come un semplice espediente del corteggiamento non sta a noi stabilirlo. Ė possibile, cioè, che Euphronios si sia realmente intrattenuto in un ginnasio frequentato dai ragazzi di cui si circonda nello psykter sopra descritto, compreso Leagros, senza che tuttavia sia accaduto nulla di compromettente fra i due $\mathrm{e}^{41} \mathrm{o}$ che ciò abbia implicato legami di amicizia con gli altri.

Non possiamo considerare la pittura vascolare attica come una sorta di fotoricordo di momenti condivisi, dimenticando il valore paradigmatico del linguaggio per immagini. Al contempo, tuttavia, non si può neppure negare il suo aggancio con la

124-132 che considera i vasi sovversivi, poiché tradurrebbero le aspirazioni all’isonomia da parte dei banausoi.

${ }^{34}$ Si pensi alla figura del mercante Sostratos, ricordata da Erodoto per l'enorme fortuna accumulata grazie al commercio marittimo in Occidente (Hdt. IV 152) oppure ad Onetorides, personaggio dagli oscuri natali che, in virtù della prosperità economica raggiunta, viene eletto arconte nel 527/26 a.C. Cfr. KANTZIOS 2005, pp. 236-237.

${ }^{35}$ I nomi attestati sono quelli di Andokides e Mnesikles, Kriton e Skythes, Phrynos e Aristogeitos, Nearchos, Peikon, Aischines, Euphronios, Smikros e figli, Xenokles, Archeneides, Brygos. Cfr. RAUBITSCHEK 1949, pp. 384-392; WEBSTER 1972, pp. 2-8.

${ }^{36}$ Si vedano le fonti citate alla nota 31.

${ }^{37}$ NEER 2002, p. 101, con bibliografia.

${ }^{38}$ Una serie di studi sottolinea come gli antroponimi relativi a certi gruppi familiari compaiano ripetutamente nei vasi prodotti dalle stesse officine. Si vedano, a tale proposito: SHAPIRO 1980, pp. 289-293; ID. 1982, pp. 69-73; ID. 1983, pp. 305-310.

${ }^{39}$ Cfr. LANGLOTZ 1920, pp. 48-54; RoBinSON - FLUCK 1937, pp. 132-136; RAUBiTSCHEK 1939, pp. 155-164; DAVIES 1971, pp. 90-92; FRANCIS - ViCKERS 1981, pp. 97-136; TÖLlE KASTENBEIN 1983, pp. 573-584; BOARDMAN 1992, pp. 45-50; PARKER 1994, pp. 365-373.

${ }^{40}$ Lenigrad - Ermitage 644, da Cerveteri; tav. 1, n. 24. Il vaso richiama l'hydria di Phintias, dove due etere giocano al kottabos acclamando Euthymides (ARV² 23.7,1608, 1620, Para 323, Add² 155; NEER 2002, pp. 133-134, P5 e I4).

${ }^{41}$ Che certe passioni, per così dire "non autorizzate", scoccassero nonostante il divieto della consuetudine è dimostrato dall'altare dedicato dai meteci ad Anteros sull'Acropoli ateniese, a memoria dell’infausto amore del meteco Melete per il giovane aristocratico Timagora. Cfr. Paus. I 30, 1. 
realtà ${ }^{42}$. Sostanzialmente, va superata la dicotomia ermeneutica fra rappresentazione simbolica e riproduzione del reale.

I vasi dei Pionieri riflettono la complessità di un momento storico estremamente magmatico, testimoniando la consapevole acquisizione di specifiche pratiche sociali, da parte di soggetti prima esclusi dall'intrattenimento d'élite e dalla paideia. Se traducono un'aspirazione, può essere solo quella al riconoscimento ufficiale di uno status già raggiunto di fatto ${ }^{43}$. Ciò, ad ulteriore riprova di come la "rivoluzione clistenica del 507 a.C. o la netta cesura fra tirannide e democrazia siano soltanto invenzioni proiettate dalle fonti di età classica sulle coscienze moderne ${ }^{44}$.

\section{Gli Anacreontic Vases}

Dal punto di vista culturale, i manufatti appena discussi costituiscono il côté complementare dei contemporanei Anacreontic vases, dove il dispiego di lusso raggiunge vertici che materializzano alla perfezione quell'habrosyne amata da Saffo e celebrata dalla lirica di Alceo, Mimnermo, Anacreonte ${ }^{45}$. Di nuovo, l'attenta lettura di quello che all'apparenza sembra classificabile come fenomeno di costume svela risvolti sociali di classe e appartenenza facilmente riconoscibili.

Gli Anacreontic vases compaiono attorno al 530 a.C. ed esattamente come gli psykteres continuano ad essere prodotti soltanto fino al 470 a.C. ${ }^{46}$. La denominazione si deve alla presenza dell'iscrizione "Anakreon" su almeno tre esemplari, accanto ad uno dei personaggi raffigurati ${ }^{47}$. Le immagini che li caratterizzano mostrano figure apparentemente abbigliate alla maniera muliebre, ma inequivocabilmente virili e, in realtà, vestite secondo dettami ispirati al gusto ionico di ascendenza lidia: lungo chitone ionico e himation, mitrai sul capo, orecchini, stivaletti o calzature particolari. I personaggi, generalmente colti nell'atto di suonare o danzare compostamente, recano spesso in mano un ombrellino parasole o il barbiton $^{48}[$ fig. 3].

Date le coincidenze cronologiche con il fenomeno degli psykteres, appare fondamentale chiarire le ragioni del diffondersi e dello scemare di quei valori di cui

\footnotetext{
${ }^{42}$ Esemplificative sono le scene di partenza per la guerra, da non collocare certo nella categoria spaziotemporale, ma profondamente legate alla realtà contemporanea.

${ }^{43}$ Chi poteva impedir loro l'accesso al ginnasio (ad Atene quello del Cinosarge è addirittura dedicato ai nothoi!), di organizzare momenti conviviali e atteggiarsi come veri aristocratici, se pure la cosa poteva infastidire gli aristoi di vecchia data?

${ }^{44}$ Del tutto superata, ormai, è la prospettiva marxista che vede nella lotta di classe e nella progressiva acquisizione di potere da parte del popolo i fondamenti del fenomeno urbano prima e del risvolto “democratico poi. Cfr. LAVELlE 2005, pp. vii-viii, 3, 155-167; DuPLOUY 2006, pp. 12-35.

${ }^{45}$ Raccolta delle fonti in KURKE 1992, pp. 91-120.

46 Nell'ambito dell'amplia bibliografia sul tema si vedano: GREIFENHAGEN 1961, pp. 117-133; IMMERWAHR 1965, pp. 152-154; DE VRIES 1973, pp. 32-39; MCINTOSH SNYDER 1974, pp. 244-246; SlATER 1978, pp. 185-194; DAVIES 1981, pp. 288-299; FrONTISI DUCROUX - LiSSARRAGUE 1990, pp. 211-256; PRICE 1990, pp. 133-160; KURKE 1992, pp. 91-120; ROSENMEYER 1992, pp. 29-33; MiLLER 1999, pp. 223-253; NEER 2002, pp. 18-23; KANTZIOS 2005, pp. 227-245.

${ }^{47}$ La coppa di Oltos, Londra E18; la lekythos del Pittore di Gales, Siracusa 26967; il frammentario cratere a calice del Pittore di Kleophrades, Copenhagen 13365. Che si tratti o meno di ritratti del poeta e dei suoi compagni (la letteratura scientifica sopra citata alla nota 46 si divide a tale proposito), ai fini della nostra esegesi, non cambia. L'antroponimo, d'altra parte, corre accanto al barbiton, non al protagonista della scena, alludendo alla possibilità che le immagini evochino semplicemente un contesto simbolico di ideali. Va ricordato, a tale proposito, anche uno psykter dove compare il nome di Kydias di Ermione accanto ad un barbiton (tav. 1, n. 28), dimostrando come ad entrare in gioco nel frangente storico sia genericamente tutto il kosmos ionico di valori, non solo Anacreonte che ne diviene probabilmente un portavoce privilegiato.

${ }^{48}$ MiLleR 1992, pp. 91-105.
} 
Anacreonte, presente ad Atene attorno al 520 a.C. ${ }^{49}$, doveva in qualche modo essere divenuto simbolo.

Già da tempo sono stati messi in luce sia gli stretti legami intercorsi fra l'Atene di Pisistrato e la Samo policratea, sia la propaganda tirannica relativa ai temi dell'autoctonia ateniese e dei suoi ancestrali vincoli etnici con la stirpe ionica ${ }^{50}$. Sembrano essere i tiranni ateniesi, dunque, ad inaugurare e perseguire una politica di connubio con il vicino Oriente, che trova riscontro anche dal punto di vista del costume $^{51}$. Eppure, quella che per decenni è stata definita "cultura della tirannide" non può essere distinta dalla cultura aristocratica contemporanea. Pisistrato è soltanto un primus inter pares, un'espressione estremamente visibile del proprio ambiente culturale con il quale intrattiene naturalmente rapporti strettissimi ${ }^{52}$. Non c'è ambiguità politica nella reiterata connivenza dei tiranni ateniesi con i membri delle famiglie rivali ${ }^{53}$, né contraddizione nel propagandare il sistema di valori aristocratico, manipolandone i temi chiave a proprio vantaggio ${ }^{54}$. I grandi protagonisti dell'Atene arcaica sono espressione di un medesimo contesto sociale e perseguono all'unisono l'unico obiettivo del potere personale.

Lo stile e gli oggetti orientali sono soltanto strategie individuali di valorizzazione sociale, la veste formale scelta dalle aristocrazie ateniesi per autodefinirsi $^{55}$, laddove il richiamo più o meno consapevole alla Grecia ionica come fonte e origine del privilegio di nascita diviene occasionalmente un aspetto del tutto relativo. Il verificarsi dei fenomeni descritti, all'indomani delle riforme che andranno definitivamente a scardinare il potere politico delle ancestrali phylai ioniche, appare quindi come un fatto quasi scontato. Mentre i banausoi si confondono, perlomeno sul piano iconografico, con i kalokagathoi, questi ultimi cercano di consolidare la propria incerta posizione politica, ottenuta in alcuni casi grazie al consenso dei ceti sociali inferiori, proprio attraverso l'esasperazione delle tradizioni aristocratiche più radicali. Ma in questo non può esserci distinzione fra sostenitori della tirannide o meno, se pure sono i tiranni stessi ad incentivare una politica di strette relazioni con l'ambiente ionico e orientale.

\footnotetext{
49 Anancreonte è generalmente considerato un membro della corte pisistratide: Vox 1990, pp. 7-8; KANTZIOS 2005, pp. 227-245. Si vedano, tuttavia, le acute considerazioni di SLINGS 2000, pp. 57-77 che negando l'esistenza di una vera e propria corte dei tiranni, ridimensiona il legame del poeta con Ipparco, evitando di appiattire la sua fase ateniese su quella presso Policrate.

${ }^{50}$ Emblematica la purificazione di Delo, la sede di Apollo, padre della stirpe Ionica. Cfr. SHAPIRO 1981, pp. 138-141.

${ }^{51}$ A ragione si ritiene che il passaggio delle donne ateniesi dal peplo dorico al chitone ionico deve aver avuto un significato culturale che va ben al di là dell'aneddotica del racconto erodoteo (Hdt. V 87-88). Cfr. BIEBER 1967, p. 28; SHAPIRO 1981, p. 138. Come sintetizza acutamente TORELLi 1978, p. 720, è alla tirannide che si deve nel VI sec. a.C. "la spinta in senso ionizzante della cultura, espressione, non occorre ripetere, di espressioni conscie ed inconscie di vario livello e motivazione a seconda delle diverse situazioni, alla habrosyne orientale".

52 BLOK 2000, pp. 31-32; BOERSMA 2000, p. 49-56; SANCISI WEERDENBURG 2000a, pp. 1-15; SANCISI WEERDENBURG 2000b, pp. 79-106; SLINGS 2000, pp. 60-66.

${ }^{53}$ Sappiamo che Pisistrato, ad un certo punto, richiama dall'esilio Cimone, le cui terre non sono mai state confiscate (Hdt. VI 103.2); conosciamo anche la sua politica matrimoniale che lo lega ai rivali Alcmeonidi e gli permette la scalata al potere (Arist. Ath. Pol. XVI). Le liste degli arconti, inoltre, dimostrano come Ippia e Ipparco utilizzassero l'arcontato come strumento di controllo e mediazione con l'enturage aristocratico. Nel 525/24 a.C. diviene arconte Clistene, figlio dell'Alcmeonide Megacle, mentre l'anno seguente è nominato Milziade, figlio di Cimone e nipote del Milziade fondatore del Chersoneso Tracico. Cfr. GhinatTi 1970, pp. 89-113; LEWIS 1988, pp. 287-302; KANTZIOs 2005, p. 236.

${ }^{54}$ Significative considerazioni in MARCHIANDI 2003, pp. 65-66.

${ }^{55}$ Duplouy 2006, pp. 278-279; HALl 2007, pp. 267-270.
} 
Sono le fonti, del resto, a confermare una simile lettura del fenomeno. Tucidide ricorda come ai suoi tempi, i vecchi dei ceti più elevati, gli eudaimones, avessero abbandonato di recente l'uso di chitoni di lino e dell'acconciatura maschile con lunghi capelli annodati in crocchie e fermagli d'oro a forma di cicala, prima adottati in segno di lusso. Lo storico contrappone poi l'inclinazione all'ostentazione degli Ateniesi all'austerità degli Spartani, i primi ad adottare una veste semplice come quella indossata dai suoi contemporanei ${ }^{56}$. Alcuni passi di Aristofane confermano come le espressioni di costume in questione non siano da identificare con una fazione specifica dell'Atene arcaica, ma costituiscano piuttosto l'emblema di un momento storico, di un sistema di valori comunemente condiviso ad alto livello ed evidentemente superato con le guerre persiane ${ }^{57}$.

Considerato quanto premesso, se nello stile cosiddetto anacreontico dobbiamo scorgere gli ideali propagandati da tutta l'aristocrazia ateniese, tirannide compresa, nel suo tramonto è da vedere non il comporsi definitivo degli antagonismi politici che fanno da perno alla storia d'Atene arcaica, ma l'esaurirsi della funzione svolta dai segni e dai modelli culturali selezionati dalle aristocrazie arcaiche per definire se stesse. Un destino simile tocca ai temi della paideia omoerotica, che non nascono certo con Pisistrato, ma nella fase della tirannide raggiungono l'apice della loro diffusione nella pittura vascolare, per poi scemare e lasciare il posto a scene ispirate a rapporti eterosessuali sullo scorcio del VI sec. a.C. ${ }^{58}$.

Nel periodo che segue le guerre persiane, al modello etico ispirato all'habrosyne orientale si va sostituendo quello isonomico lacedemone. Soltanto ciò che sarà capace di inscriversi nella nuova cornice "democratica sarà destinato a durare nel tempo. La "democrazia, di cui le leggi suntuarie sono figlie, sarà la nuova formula adottata dagli aristoi per mantenere l'egemonia sui propri rivali ${ }^{59}$.

\section{Funzione simbolica e significato dello psykter}

Giunge ormai momento di chiederci se e in quale maniera lo psykter possa essere assimilato ai valori che ispirano lo "stile anacreontico. Che oltre al medesimo arco cronologico il vaso e la "moda in questione possano avere altro in comune è suggerito da un'anfora a figure nere [fig. 4], firmata attorno al 530 a.C. dal vasaio Xenokles, anche noto come dedicante sull'Acropoli, e dall'altrimenti ignoto pittore Kleisophos $^{60}$. Il vaso, che non ha sinora ricevuto la meritata attenzione, mostra l'impiego dello psykter nell'ambito di un simposio dove i commensali indossano

56 Thuc. I 6, 3: "Furono gli Ateniesi i primi ad abbandonare le armi e, vivendo liberamente, ad adottare modi più raffinati. E non è passato molto tempo da quando in Atene i più vecchi tra i ricchi hanno abbandonato l'usanza, che era segno di lusso, di portare chitoni di lino e legare il nodo dei capelli inserendovi cicale d'oro. Da qui questa moda passò e rimase a lungo, per la loro parentela con gli Ateniesi, tra gli Ioni, presso i vecchi”. La testimonianza appare significativa, sebbene consideri erroneamente la moda ionica come un'invenzione ateniese.

${ }^{57}$ Cfr. Arist. Equites v. 1331, dove il Demos ringiovanito appare in scena con le cicale d'oro nei capelli; Nubes vv. 984-986, dove il "Discorso peggiore disprezza i valori d'un tempo, definendoli "roba dei tempi delle spille a forma di cicala"; Ath. XII 512 B-C.

${ }^{58}$ SHAPIRO 1981, pp. 133-143.

59 DuplouY 2006, pp. 254-255. La riforma di Clistene opera nella medesima direzione: depauperando le antiche phylai ioniche del potere politico, non vuole genericamente colpire l'aristocrazia di lignaggio, cui il suo stesso promotore appartiene, ma coloro che nel lignaggio continuano a fondare i presupposti del proprio statuto.

${ }^{60}$ Atene, Museo Nazionale CC691; tav. 2, n. 1. Per la dedica di Xenokles sull'Acropoli si veda: WEBSTER 1972, pp. 2-8. 
alcuni accessori tipici dello stile anacreontico. Si tratta certamente di un prodotto eccezionale, come suggerisce la duplice firma apposta dal vasaio e dal pittore. L’orgoglio dei due artigiani si deve, con tutta probabilità, proprio al messaggio veicolato dal vaso, poiché la forma non sembra avere niente di straordinario ${ }^{61}$. L'eleganza e la misura che generalmente contraddistinguono i simposiasti "anacreontici sembrano qui aver abbandonato del tutto i protagonisti della scena. I cinque uomini sono palesemente ubriachi e ormai nudi, pur indossando tutti la mitra e tre di essi gli stivaletti. Uno beve seduto a terra, un altro è addirittura raffigurato in braccio ad un compagno nell'atto di urinare sopra ad un altro commensale adagiato a terra sotto di esso. Al centro della scena, un giovane coppiere è affaccendato presso un enorme cratere a calice decorato da un cavallo e all'interno del vaso galleggia uno psykter. Cittadini ateniesi d'altissimo rango - sul cratere troneggia il simbolo per eccellenza degli hippeis - sono rappresentati alla guisa di satiri: l'intento è chiaramente parodico. Esattamente come nei vasi dei Pionieri e nelle scene di simposio "anacreontico, ad essere in scena, nell'anfora contemporanea alle prime produzioni di psykteres e crateri a calice, è il dispiego di lusso. Eppure, in questo caso, tutto sembra avere una connotazione inversa a quella consueta. Non sorge alla memoria la "lydopatheia ${ }^{62}$ dei lirici arcaici, ma gli ironici attacchi all'habrosyne di Semonide e Senofane ${ }^{63}$. Dal punto di vista narrativo, l'effetto caricaturale è raggiunto attraverso la ridondanza visiva di segni, pertinenti ad una sfera di valori diametralmente opposta all'atteggiamento satirico degli protagonisti.

Il manufatto di Xenokles permette, da un lato, di identificare lo psykter come un vaso d'uso non comune, poiché viene utilizzato insieme al resto come simbolo di un ben preciso entourage sociale, un aspetto del tutto in linea con una produzione che può essere definita d'altissima qualità. D'altro canto, si fa testimone di un atteggiamento quasi dissacrante, da parte di alcuni artigiani, nei confronti degli hippeis $^{64}$.

Cerchiamo ora di definire meglio la presenza dello psykter in una simile dimensione. Possiamo immediatamente escludere la possibilità che il vaso facesse parte del "servizio anacreontico per il simposio. Sappiamo, infatti, che il vaso galleggiante a forma di fungo nasce nelle officine ateniesi e non sembra avere precedenti nella tradizione ceramica. Le anfore-psykteres, sebbene ugualmente destinate a raffreddare il vino, presentano una morfologia del tutto differente che non occupa certo la medesima parte nello spazio del simposio. Tali anfore precedono di poco l'invenzione dello psykter e vengono introdotte in Attica dalla Tracia e dalla Beozia $^{65}$. Ciò sconsiglia di ipotizzare un'eventuale importazione dalla Ionia dell'uso di bere vino freddo nel simposio. La presenza dello psykter nelle scene vascolari, infine, non sembra assolutamente circoscrivibile ai soli contesti anacreontici. Dobbiamo pertanto concludere che l'impiego del vaso sia solo genericamente da collocare molto in alto nella scala sociale.

\footnotetext{
${ }^{61}$ Il pezzo non è certo paragonabile alla rivoluzionaria morfologia del cratere François!

${ }^{62}$ Il termine è un neologismo di NEER 2002, p. 19. Anacreonte si dichiara “lydopatheis” (fr. 136/148 $P M G)$.

${ }^{63}$ Sem. fr. 7 W, 57-70; Xenoph. frr. 2 D-K, 3 D-K. Cfr. KuRKE 1992, pp. 94-95.

${ }^{64}$ Sarà da valutare, a tale proposito, anche il nome del ceramista "Xenokles", che potrebbe alludere ad uno straniero, un non cittadino. Cfr. CHANTRAINE 1968, s.v. xenos (derivati antroponimici); TRAILL 2004, pp. 362-363, n. 731880.

${ }^{65}$ Sull'anfora-psykter: KARO 1899, pp. 141-143; VIERNEISEL 1990, p. 261; SCHREIBER 1999, pp. 221223. L'unica anfora-psykter ateniese conosciuta è stata significativamente realizzata da Lydos: BOARDMAN 1974, pp. 52-54 fig. 66.
} 
La nostra analisi del problema dovrebbe fermarsi qui se dovessimo procedere su binari esclusivamente morfologici o di contesto immediato, ma allargando la prospettiva alle funzioni assolte dallo psykter sembra possibile andare oltre. Se non sono la forma o le immagini da essa veicolate a fornire una spiegazione logica alla sua palese condanna attorno al 470 a.C., infatti, sarà da indagare il significato simbolico dello psykter nell' arco di tempo che lo vede in uso.

In modo forse prevedibile, le fonti sono piuttosto chiare nel collocare il consumo di vino freddo o addirittura neve in contesti simposiaci di altissimo livello ${ }^{66}$. La ragione di ciò riposa nella banale considerazione che nei caldi paesi mediterranei come l'Ellade la neve è un fenomeno raro e i costi del suo approvvigionamento devono essere stati inimmaginabili. Così, Euticle dice che "Chi per primo sa se la neve si può comperare, / quello per primo deve mangiare senz'altro favi di miele" 67 , alludendo al fatto che la neve, come certi mieli pregiati, è reperibile solo sulle vette più elevate ${ }^{68}$. Anassandride, descrivendo nel Protesilao il sontuosissimo banchetto offerto da Cati re di Tracia, in occasione delle nozze di Ificrate con sua figlia, ricorda tra i doni nuziali una brocca di neve ${ }^{69}$. Ateneo evoca i passi di Macone dove la dotta etera Gnatea riceve in dono neve da un amante siriano ${ }^{70}$. A destare particolare interesse è infine l'epigramma improvvisato da Simonide $^{71}$ in occasione di un incontro conviviale estivo, mentre i coppieri mescolano neve al vino per gli altri ma non per lui: 'L'avviluppò, una volta, intorno ai fianchi d'Olimpo / un violento Borea, abbattendosi dalla Tracia; / e di uomini senza mantello morse il cuore, ma fu piegata / la sua cintura, avvolgendosi intorno alla terra di Pieria; / una sola parte anche di essa mi si versi. Non sta bene / levare un brindisi di caldo vino ad un amico"72.

Diversi passi di Ateneo dimostrano, tuttavia, come a subire un contraccolpo dopo le guerre persiane non sia il semplice consumo di neve ${ }^{73}$, ma evidentemente la sua ostentazione attraverso l'impiego di un oggetto ricercato e futile. Ad essere incriminata, pertanto, non è la funzione primaria dello psykter in qualità di contenitore, ma quella, solo per noi secondaria, afferente al campo della semiotica, che inscena le potenzialità percettive dell'oggetto.

Come già richiamato, grazie alla sua peculiare forma dotata di coperchio, lo psykter ha certo il vantaggio di conservare la neve a lungo e può essere ripetutamente

\footnotetext{
${ }^{66}$ La pratica di conservare neve in ghiacciaie sotterranee per mescolarla al vino o refrigerare l'acqua d'estate, nelle case d'alto rango, è ben documentata da Ateneo. Cfr. Ath. II 71e, III 123a, e-f, III 125a. Cfr. Xen. Memorabilia II, 1.30.

${ }^{67}$ Fr. I KASSEL-AuSTIN apud Ath. III 124d. L'espressione “mangiare favi di miele” letteralmente allude alla pratica di masticare a lungo il residuo del favo in cui era conservato il miele, per separarlo dalla cera che infine veniva sputata. Cfr. GIUMAN 2008, p. 72.

${ }^{68}$ Cfr. Pl. N.H. XI 13.

${ }^{69}$ Il passo di Anassandride (fr. 2 Kassel-Austin), riportato nei Deipnopsofisti (Ath. IV 131a-d), mette a confronto la sontuosità barbarica del banchetto nuziale tracio con la raffinata misura dei convivi attici. Anche in tale frangente, pertanto, al consumo di neve fa da cornice uno sfoggio di lusso estremo, percepito come abnorme da chi è abituato alla frugalità attica.

${ }^{70}$ Ath. XIII 579. Al di là del disprezzo del dono da parte della donna, va sottolineata la provenienza medio-orientale dell'amante.

${ }^{71}$ Anche il poeta di Ceo frequenta la cerchia degli intellettuali che ruotano attorno ad Ipparco e le sue poesie mostrano un stretta affinità tematica con quelle di Anacreonte. Cfr. Ps.Plato Hipp. 228c; Arist. Const. Ath. XVIII; KANTZIOs 2005, p. 235 nota 37; PolterA 2008, pp. 6-8. Altro poeta ionico, accolto in quegli anni ad Atene, è Kydias di Ermione. Cfr. SHAPIRO 1982, p. 72.

${ }^{72}$ Callistr. FGrHist 348 F 3; Sim. fr. 25 WEST = Epigr. 88 PAGE. La traduzione riportata nel testo è di L. CANFORA (Ateneo. I Deipnosofisti. Il dotti a banchetto, vol. I libri I-V, Roma 2001, Ath. 125c-d). Il soggetto dell'epigramma è chiaramente la neve dell’Olimpo.

73 Cfr. nota 66.
} 
rimboccato $^{74}$. Rispetto all'impiego tradizionale dell'acqua fredda mescolata direttamente al vino nel cratere, lo strano vaso offre la possibilità di attingere neve o acqua appena sciolta in modo graduale, per rinfrescare, eventualmente, anche il liquido già versato nelle coppe, come forse accadeva nella circostanza recitata dal poeta di Ceo. Ma soprattutto, la presenza di uno psykter galleggiante nel cratere rende immediatamente tangibile dal punto di vista visivo l'impiego di neve. Ciò vale sia per i convitati reali d'un tempo, sia per lo spettatore - antico e moderno - di una scena vascolare. Il vaso si propone di per sé come elemento puramente accessorio, un vezzo colorato di immagini significative, inventato per gratificare il palato, incuriosire e allietare lo spettatore.

Il consumo di neve in occasioni conviviali attraverso lo psykter, pertanto, doveva essere percepito dai contemporanei come un lusso estremo, divenendo facile emblema di quell'habrosyne perseguita dalle aristocrazie ateniesi di età arcaica e trasformata dagli anni successivi alle guerre persiane nella negazione stessa di grecità. Nel V sec. a.C., un intero campo semantico slitta infatti dal termine habrosyne a quello di tryphe. Ciò è ben sintetizzato dal risvolto del concetto stesso di habrosyne, che con le guerre persiane assume un'irrevocabile connotazione negativa ${ }^{75}$, afferente ad una sfera di valori che può costare l'ostracismo.

Nel 507 a.C. le richieste di aiuto alla Persia, perpetrate da Clistene per contrastare la coalizione di Isagora appoggiata da Sparta ${ }^{76}$, sono accolte ad Atene come un fatto naturale. Nel 496-95 a.C., all'indomani della rivolta ionica e in pieno regime democratico, Ipparco figlio di Carmo raggiunge l'arcontato proprio grazie ai suoi legami di casata con il Gran Re e alla speranza che potesse mediare con l'Oriente, evitando lo scontro. Nella primavera del 487 a.C., tuttavia, viene accusato di medismo ed è bandito da Atene ${ }^{77}$. Anche Kallias Kratiou di Alopeke, rappresentato attorno al 510 a.C. dal Pittore di Ambrosios in compagnia del poeta Kydias di Ermione, nel 485 a.C. viene ostracizzato per la medesima causa ${ }^{78}$. Diversi ostraka lo definiscono "ho medos" e uno lo raffigura addirittura con un copricapo persiano, pantaloni e scarpe a puntini ${ }^{79}$. Nella nuova dimensione "democratica, il concetto di medismo giunge a comprendere l'insieme di valori della cultura aristocratica di età arcaica, filtrato dall'accezione moralizzante di V sec. a.C. "Medizzare significa ostentare, agire in modo ormai eticamente inaccettabile ${ }^{80}$. Ancora una volta, sebbene in una logica e contrario, è l'Oriente ad essere chiamato in causa per riassumere un intero mondo, percepito nel nuovo contesto sociale come obsoleto e inadeguato. Con le guerre persiane crolla l'intero sistema di codici funzionali all'aristocrazia di età arcaica per sostanziare e definire se stessa. Così, lo psykter, espressione a pieno titolo dell'habrosyne all'indomani del conflitto con la Persia, attorno al 470 a.C. viene debitamente eliminato dalle mense e dall'immaginario collettivo ateniesi ${ }^{81}$.

\footnotetext{
${ }^{74} \mathrm{Si}$ veda la p. 12 di questo contributo.

${ }^{75}$ GHINATTI 1970, pp. 98-99, 122-124; KURKE 1992, pp. 98-114.

${ }^{76}$ Hdt. V 70-73.

${ }^{77}$ GHinATTI 1970, p. 127; HALl 2007, p. 269.

${ }^{78}$ Coppa Monaco 2614, ShapIRO 1982, pp. 69-73, fig. 1, tav. 25a; HALL 2007, pp. 269-270. Per l'accusa di “medismo, raccolta delle fonti in SIEWERT 2002, pp. 156-158.

${ }^{79}$ SHAPIRO 1982, pp. 69-73; SIEWERT 2002, p. 156 T1/46-61.

${ }^{80}$ Cfr. HALl 2007, pp. 268-270. Lo conferma il fatto che la stessa accusa viene perpetrata contro soggeti per i quali non è documentato alcun tipo di rapporto con l'Oriente. Si veda, a tale proposito, in SIEWERT 2002, pp. 84-85, T1/41, il caso di Habronichos sul quale l'A. si interroga proprio per l'assenza di elementi concreti che lascino ipotizzare una connivenza con l'elemento persiano.

${ }^{81}$ Un passo di Crisippo di Soli (apud Ath. IV 137f) conferma come agli oggetti da mensa e ai cibi stessi fosse attribuito un valore simbolico piuttosto forte. La fonte narra la reazione dei sacerdoti
} 
La diffusione dei temi anacreontici e dello psykter nelle due generazioni che segnano la fine dell'arcaismo, la successiva loro scomparsa alle soglie della classicità, vanno di pari passo nell'esprimere la parabola dei valori di un sistema culturale in crisi che riedifica se stesso su nuove basi. Il riformularsi degli equilibri sociali, che caratterizza gli anni finali del VI sec. a.C., induce le famiglie in competizione per il potere ad un'espressione del lusso sempre più ricercata, affinché lo stile di vita possa assumere quel ruolo di distinguo che il denaro e il lignaggio non sono più in grado di rappresentare. Motore del sistema è la politica di prestigio e di concentrazione della ricchezza incentivata dai tiranni, ma unanimemente perseguita da tutte le élites ateniesi. In ragione di ciò, la scomparsa dei temi oggetto di indagine non avviene con la caduta della tirannide, ma alcuni decenni più tardi. La cultura materiale dei sessant'anni che precedono le guerre persiane, ad un livello "alto, infatti, esprime un sistema di valori coscientemente condiviso da tutte le aristocrazie locali che, in diversa misura, aspirano all’assunzione del potere politico.

dell’Accademia e del Liceo nei confronti di due cuochi, per aver osato introdurre nei banchetti elementi non conformi alle norme della città. Il primo aveva portato una casseruola (lopas) proveniente da Caristo, nonostante il divieto di usare in occasioni ufficiali suppellettile importata. Il secondo, invece, aveva salato della costosa carne alla maniera del più frugale pesce. All'Accademia i sacerdoti impongono di spezzare tutte le stoviglie, mentre al Liceo il cuoco viene frustato per aver servito un piatto sconvenientemente ricercato. 


\section{Bibliografia}

\section{BIEBER 1967} BLOK 2000

M. BIEBER, Entwicklungsgeschichte der griechischen Tracht 2, Berlin 1967.

J.H. Blok, Phye's Procession: Culture, Politics and Peisistratid Rule, in H.

SANCISI WeERDENBURG (a cura di), Peisistratos and the Tyranny: a Reappraisal of the Evidence, Amsterdam 2000, pp. 17-48. BOARDMAN 1974

J. BoARDMAn, Athenian Black-figured Vases, London 1974. BOARDMAN 1977

J. BoARDman, Review: S. Drougou: Der attische Psykter, Wurzburg 1975, in «ClRev» New Series, 1977 (XXVII.2), p. 306.

BOARDMAN 1992

J. BoARdman, Kaloi and Other Names in Euphronios' Vases, in M. Cygielman et AliI (a cura di), Euphronios, Atti del Seminario Internazionale di Studi (Arezzo 27-28 maggio 1990), Firenze - Milano 1992, pp. 45-50.

BOARDMAN 2004

J. BOARDMAN, Storia dei vasi greci, Roma 2004 (tr. it).

BOERSMA 2000

J. Boersma, Peisistratos' Building Activity Reconsidered, in H. SANCISI WeErdenburg (a cura di), Peisistratos and the Tyranny: a Reappraisal of the Evidence, Amsterdam 2000, pp. 49-56.

VON BOTHMER 1961

D. von Bothmer, Newly Acquired Bronzes - Greek, Etruscan, and Roman, in «The Metropolitan Museum of Art Bulletin», New Series, 1961 (XIX.5), pp. 133-151.

VON BOTHMER 1986

D. VON Bothmer, An Archaic Red-Figured Kylix, in «The J. Paul Getty

Museum Journal» 1986 (XIV), pp. 5-20.

VON BOTHMER 1991

D. vON Bothmer (a cura di), Glories of the Past, Ancient Art from the Shelby

White and Leon Levy Collection, New York 1991.

CHANTRAINE 1968

P. ChANTRAINE, Dictionnaire étymologique de la langue grecque. Histoire des mots, Paris 1968.

CHRESTOS ET ALII 1978

G. Chrestos ET ALII, Benaki Museum, Athens: An Exhibition of Ancient Greek Art from the N.P. Goulandris Collection, Athens 1978.

COHEN 1995

D. Cohen, Law, Violence, and Community in Classical Athens, Cambridge 1995.

DAVIES 1971

J.K. DAVIES, Athenian Propertied Families 600-300 B.C., Oxford 1971.

DAVIES 1981

M. DAVIES, Artemon Transvestitus? A Query, in «Mnemosyne», Fourth Series, 1981 (XXXIV.3/4), pp. 288-299.

DOVER 1989

K.J. Dover, Greek Homosexuality, Cambridge (Mass.) 1989. 


\section{Drougou 1975}

S. Drogou, Der Attische Psykter, Würzburg 1975.

DUPLOUY 2006

A. Duploy, Le prestige des élites. Recherches sur les modes de reconnaissance sociale en Grèce entre les $X$ et $V$ siècles avant J.-C., Paris 2006.

\section{FRANK 1990}

S. FRANK, Attische Kelchkratere. Eine Untersuchung zum Zusammenspiel von Gefäßform und Bemalung, Frankfurt a. M. 1990.

FRANCIS - VICKERS 1981

E.D. FRANCIS - M. VICKERS, “Leagros Kalos”, in «PCPS», 1981 (CCVII), pp. 97-136.

FRONTISI DUCROUX - LISSARRAGUE 1990

F. Frontisi DuCROUX - F. LisSARRAGUe, From Ambiguity to Ambivalence: a Dionysiac Excursion through the "Anacreontic" Vases, in D.M. HALPERIN ET ALII (a cura di), Before Sexuality. The Construction of Erotic Experience in the Ancient Greek World, Princeton 1990, pp. 211-256.

GENTILI 1983

B. Gentili, Eros nel simposio, in M. Vetta (a cura di), Poesia e simposio nella Grecia antica. Guida storica e critica, Roma - Bari 1983, pp. 85-93.

\section{GERNET 1968}

L. GERNET, The Anthropology of Ancient Greece, Baltimore - London 1968.

\section{GHINATTI 1970}

F. GHINATTI, I gruppi politici persiani fino alle guerre persiane, Roma 1970.

\section{GIULIANI ET ALII 1991}

Giuliani et Alit (a cura di), Euphronios. Pittore ad Atene nel VI sec. a.C., Milano 1991.

GIUMAN 2008

M. Giuman, Melissa. Archeologia delle api e del miele nella Grecia antica, Roma 2008.

GILL ET ALII 1998

C. GILL ET ALII, Reciprocity in Ancient Greece, Oxford 1998.

GODART - DE CARO 2007

L. Godart - S. De CARo (a cura di), Nostoi, Capolavori Ritrovati, Roma,

Palazzo del Quirinale, Galleria di Alessandro VII, 21 dicembre 2007 - 2 marzo 2008, Roma 2007.

GRASSIGLI 1999

G.L. Grassigli, Tra moderno e antico. Per un confronto sull'iconologia archeologica, in «Ostraka», 1999 (VIII), pp. 447-468.

GREIFENHAGEN 1961

A. Greifenhagen, Lekythos des Kerberosmalers, in «Jahrbuch der Berliner Museen», 1961 (III), pp. 117-133.

HALL 2007

J.M. HALL, A History of the Archaic Greek World ca. 1200-479 BCE, Malden

- Oxford - Carlton 2007.

HUBER 1992

K. Huber, Werkstattgesellen. Zur Produktion früher Kelchkratere, in M. DENOYELLE (a cura di) Euphronios und seine Zeit. Kolloquium in Berlin 19. 20. April 1991, Berlin 1992, pp. 7-72. 
IMMERWAHR 1965

H.R. IMMERWAHR, Inscriptions on the Anacreon Krater in Copenhagen, in «AJA», 1965 (LXIX.2), pp. 152-154.

KANTZIOS 2005

I. KANTZIOS, Tyranny and the Symposion of Anacreon, in «ClJ», 2005 (C.3), pp. 227-245.

KARO 1899

G. KARO, Notes on Amasis and Ionic Black-Figured Pottery, in «JHS», 1899 (XIX), pp. 135-164.

KURKE 1992

L. KURKe, The Politics of habrosyne in Archaic Greece, in «ClAnt», 1992 (XI.1), pp. 91-120.

LA CECLA 2007

F. LA CECLA, La moda rende felici (per mezz’ora almeno), Milano 2007.

LANGLOTZ 1920

E. LANGLOTZ, Zur Zeitbestimmung der strengrotfigurigen Vasenmalerei und der gleichzeitigen Plastik, Leipzig 1920.

LAVELLE 2005

B.M. Lavelle, Fame, Money, and Power. The Rise of Peisistratos and "Democratic" Tyranny at Athens, Ann Arbor 2005.

LEWIS 1988

D. M. LEWIS, The Tyranny of the Pisistratidae, in J. BOARDMAN ET ALII (a cura

di), Persia, Greece, and the Western Mediterranean c. 525-479 B.C., Cambridge 1988, pp. 287-302.

LISSARRAGUE 1990

F. Lissarrague, Around the Krater. An Aspect of Banquet Imagery, in O.

Murray (a cura di), Sympotica. A Symposium on the Symposion, Oxford 1990, pp. 196-209.

LISSARRAGUE 2001

F. Lissarrague, Greek Vases. The Athenians and their Images, New York 2001.

LISSARRAGUE - THELAMON 1983

F. LissarRague - F. Thelamon (a cura di), Image et Ceramique Grecque, Rouen 1983.

MARCHIANDI 2003

D. MARChIANDI, L'Accademia: un capitolo trascurato dell' "Atene dei tiranni”, in «ASAA», 2003 (LXXXI.1), pp. 11-81.

MAUSS $2002^{3}$

M. MAUSs, Saggio sul dono. Forma e motivo dello scambio nelle società arcaiche, Torino $2002^{3}$ (tr. it.).

MCINTOSH SNYDER 1974

J. McIntosh SNyder, Aristophanes' Agathon as Anacreon, in «Hermes»,

MiLLeR 1992 1974 (CII.2), pp. 244-246.

M.C. Miller, The Parasol: An Oriental Status-Symbol in Late Archaic and Classical Athens, in «JHS», 1992 (CXII), pp. 91-105.

MiLler 1999

M.C. Miller, Reexamining Transvestism in Archaic and Classical Athens:

The Zewadski Stamnos, in «AJA», 1999 (CIII.2), pp. 223-253. 
MoON 1983

W. Moon (a cura di), Ancient Greek Art and Iconography, Madison 1983.

MOON - BERGE 1979

W. MoOn - L. Berge, (a cura di), Greek Vase Painting in Midwestern

Collections, Chicago 1979.

MOORE - PHILIPPIDES 1986

M.B. MoORE - M.Z.P. PhILIPPIDEs, The Athenian Agora. Volume XXIII. Attic

Black-figured Pottery, Princeton 1986.

NEER 2002

R.T. Neer, Style and Politics in Athenian Vase-Painting. The Craft of Democracy, ca. 530-460 B.C.E., Cambridge 2002.

PARKER 1994

V. PARKer, Zur absoluten Datierung des Leagros Kalos und der 'Leagros-

Gruppe', in «AA», 1994, pp. 365-373.

PHILIPPAKI 1967

B. PHILIPPAKI, The Attic Stamnos, Oxford 1967.

POLTERA 2008

O. PolterA, Simonides lyricus. Testimonia und Fragmenta. Einleitung, kritische Ausgabe, Übersetzung und Kommentar, Basel 2008.

PRICE 1990

S.D. PRICE, Anacreontic Vases Reconsidered, in «GrRBS», 1990 (XXXI.2), pp. 133-160.

RADFORD 1915

E. RADFORD, Euphronios and His Colleagues, in «JHS», 1915 (XXXV), pp. 107-139.

RAUBITSCHEK 1939

A. RAUBITSCHEK , “Leagros”, in «Hesperia», 1939 (VIII), pp. 155-164.

RAUBITSCHEK 1949

A. E. RaubitscheK, Dedications from the Athenian Akropolis, Cambridge Mass. 1949, pp. 384-392.

ROBERTSON 1987

M. Robertson, Greek, Etruscan and Roman Vases in the Lady Lever Art Gallery, Port Sunlight (Liverpool) 1987.

ROBERTSON 1996

M. Robertson, The Art of Vase-painting in Classical Athens, Cambridge 1996.

ROBINSON - FLUCK 1937

D.M. RoBinSON - E.J. FLUCK, A Study of the Greek Love-Names Including a Discussion of Paederasty and a Prosopographia, Baltimore 1937.

ROSENMEYER 1992

P.A. Rosenmeyer, The Poetics of Imitation and the Anacreontic Tradition, Cambridge 1992.

SANCISI WEERDENBURG 2000a

H. SANCISI WeERdenBurg, The Tyranny of Peisistratos, in H. SANCISI WeERDENBurg (a cura di), Peisistratos and the Tyranny: a Reappraisal of the Evidence, Amsterdam 2000, pp. 1-15.

SANCISI WEERDENBURG 2000b

H. SAnCisi WeErdenburg, Cultural Politics and Chronology, in H. SANCisi WeERDENBURG (a cura di), Peisistratos and the Tyranny: a Reappraisal of the Evidence, Amsterdam 2000, pp. 79-106. 


\section{SCHREIBER 1999}

T. SCHREIBER, Athenian Vase Construction. A Potter's Analysis, Santa Monica (California) 1999.

SCHEIBLER 1995

I. ScheIBler, Il vaso in Grecia. Produzione, commercio e uso degli antichi vasi in terracotta, Milano 1995.

SCHEIBLER 2001

I. SCHEIBLER, Psykter, in «Neue Pauly», 2001 (X), p. 523.

SEGRE REINACH 2005

S. SEGRE REINACH, La moda. Un'introduzione, Roma - Bari 2005.

SHAPIRO 1980

H.A. SHAPIRO, Hippokrates Son of Anaxileos, in «Hesperia», 1980 (XLIX.3), pp. 289-293.

SHAPIRO 1981

H.A. ShapIRO, Courtship Scenes in Attic Vase-Painting, in «AJA», 1981 (CXXXV.2), pp. 133-143.

SHAPIRO 1982

H.A. SHAPIRO, Kallias Kratiou Alopekethen, in «Hesperia», 1982 (LI.1), pp. 69-73.

SHAPIRO 1983

H.A. SHAPIRO, Epilykos Kalos, in «Hesperia», 1983 (LII.3), pp. 305-310.

SHAPIRO 1985

H.A. SHAPIRO, Attisch rotfiguriger Kelchkrater, in «AA», 1985, pp. 261-264.

SIEWERT 2002

P. SIEWERT (a cura di), Ostrakismos-Testimonien I. Die Zeugnisse antiker Autoren, der Inschriften und Ostraka über das athenische Scherbenbericht aus vorhellenistischer Zeit, «Historia Einzelschriften» H. CLV, Stuttgart 2002.

SISTO 2006

M.A. Sisto, Forma e decorazione figurata dello stamnos dalla Grecia alla Magna Grecia, in F. GIUDICE - R. PANVINI (a cura di), Il greco, il barbaro e la ceramica attica. Immaginario del diverso, processi di scambio e autorappresentazione degli indigeni, 3, Atti del convegno internazionale di studi, 14 - 19 maggio 2001. Catania - Caltanissetta - Gela - Camarina Vittoria - Siracusa, Roma 2006, pp. 151-163.

SLATER 1978

W.J. Slater, Artemon and Anacreon: No Text Without Context, in «Phoenix», 1978 (XXXII.3), pp. 185-194.

SLINGS 2000

S.R. SLINGS, Literature in Athens, 566-510 BC, in H. SANCISI WEERDENBURG (a cura di), Peisistratos and the Tyranny: a Reappraisal of the Evidence, Amsterdam 2000, pp. 57-77.

STAMPOLIDIS - PALARMA 2000

N. Stampolidis - L. Parlama (a cura di), Athens, The City Beneath the City, Antiquities from the Metropolitan Railway Excavations, Athens 2000.

STEINER 1998

D. STEINER, Moving Images: Fifth-Century Victory Monuments and the

Athlete's Allure, in «ClAnt», XVII.1, 1998, pp. 123-150.

STEINER 2007

A. STEINER, Reading Greek Vases, Cambridge 2007. 
TÖLLE KASTENBEIN 1983

R. TÖLLE KASTENBEIN, Bemerkungen zur absoluten Chronologie spätarchaischer und frühklassischer Denkmäler Athens, in «AA», 1983 (LXLVIII), pp. 573-584.

TORELLI 1978

M. TORELLI, La cultura artistica dell'età arcaica, in R. BIANCHI BANDINELLI

(a cura di), Storia e civiltà dei Greci. Origini e sviluppo della città.

L'arcaismo, Milano 1978, pp. 645-720.

TRAILL 2004

J.S. TRAiLL, Persons of Ancient Athens vol. 13, Toronto 2004.

VETTA 1983

M. Vetta (a cura di), Poesia e simposio nella Grecia antica. Guida storica e critica, Roma - Bari 1983.

VIERNEISEL 1990

K. VIERNEISEL, Psykter für kühlen Wein, in K. VIERNEISEL - B. KAESER (a Vox 1990

cura di), Kunst der Schale. Kultur des Trinkens, München 1990.

DE VRIES 1973

K. DE VRIES, East Meet West at Dinner, Expedition, summer 1973, pp. 32-39.

WEBSTER 1972

T.B.L. Webster, Potter and Patron in Classical Athens, London 1972. WINKLER 1990

J.J. WinkLER, The Constraints of Desire: The Anthropology of Sex and Gender in Ancient Greece, New York 1990. 
Tav. 1 Produzione, cronologia e iconografia degli psykteres.

\begin{tabular}{|c|c|c|c|c|c|c|c|}
\hline $\mathbf{N}$. & TECNICA & CERAMISTA/PITTORE & DATAZIONE & ICONOGRAFIA & ISCRIZIONI & N. INV. & BIBLIOGRAFIA \\
\hline 1 & FN & Gruppo del Cigno & 540 a.C. & Cigni & & $\begin{array}{c}\text { Atene, Museo dell'arte cicladica e } \\
\text { arcaica } 11 \\
\end{array}$ & Chrestos et alii 1978, 240.113 \\
\hline 2 & FN & Gruppo del Cigno & 540 a.C. & & & Atene, M. Vlasto 332151 & $\begin{array}{c}A B V \text { 658.125; Drougou 1975, } \\
\text { 19 B2 }\end{array}$ \\
\hline 3 & FN & Gruppo del Cigno & 540 a.C. & Decorazione floreale, foglie & & $\begin{array}{c}\text { Berkeley (CA), Phoebe Apperson } \\
\text { Hearst Museum of Anthropology } \\
\text { D194 }\end{array}$ & $\begin{array}{l}A B V \text { 658.126; } A d d^{2} 147 ; \\
\text { Drougou 1975, 18-19 B1 }\end{array}$ \\
\hline 4 & FN & $\begin{array}{l}\text { Nikostenes / P. di } \\
\text { Nikosthenes }\end{array}$ & 530-520 a.C. & Gigantomachia & & $\begin{array}{c}\text { Houston, Texas, de Menil } \\
\text { Collection } 70.53 \\
\end{array}$ & Drougou 1975, 13 A7. \\
\hline 5 & FN & P. di Lisyppides & 530-500 a.C. & $\begin{array}{c}\text { Quadriga di prospetto fra } \\
\text { guerrieri e arcieri }\end{array}$ & & $\begin{array}{c}\text { Malibu (CA), Paul Getty } \\
\text { Museum 96.AE.94 }\end{array}$ & $\begin{array}{c}\text { Barbara and Lawrence } \\
\text { Fleischman 1994, 87-88, n.36 }\end{array}$ \\
\hline 6 & FN & & 530-500 a.C. & $\begin{array}{l}\text { Dioniso recumbente a terra e } \\
\text { sul mulo, fra satiri e menadi }\end{array}$ & & Parigi, Louvre CP12296 & Drougou 1975, 14 A10, tav. 8.2 \\
\hline 7 & FN & P. di Antimenes & 525-500 a.C. & $\begin{array}{c}\text { Dioniso assiso fra satiri e } \\
\text { menadi, Efesto (?) sul mulo }\end{array}$ & & $\begin{array}{c}\text { Coll. Port Sunlight, Lady Lever } \\
\text { Art Gallery } 2138\end{array}$ & $\begin{array}{c}\text { Drougou 1975, } 13 \text { A8; } \\
\text { Robertson 1987, tavv.18-19 } \\
\end{array}$ \\
\hline 8 & FR & Gruppo di Pezzino & 525-500 a.C. & Atleti & Iscriz. "nonsense" & Zurigo, Univ. 4039 & $\begin{array}{c}\text { Drougou 1975, } 17 \text { A27, } 94 \text { e n. } \\
\text { 316; Bothmer 1986, fig. 6a-d }\end{array}$ \\
\hline 9 & FN & Maniera del P. di Antimenes & 525-500 a.C. & Partenza di guerrieri & & $\begin{array}{l}\text { Lipsia, Antikenmuseum Karl } \\
\text { Marx Universität Leipzig T367 }\end{array}$ & $\begin{array}{c}\text { CVA 2, 29-30, tavv. 27.1-2, } \\
\text { 28.3-4; Drougou 1975, } 12 \text { A2 }\end{array}$ \\
\hline 10 & FN & Maniera del P. di Antimenes & 525-500 a.C. & Partenza del guerriero & & Parigi, Louvre F319 & $\begin{array}{l}\text { CVA 8, tavv. 73.2-3.7, 74.1.4; } \\
\text { Drougou 1975, } 13 \text { A3, tav. 7.1 }\end{array}$ \\
\hline 11 & FN & Maniera del P. di Antimenes & 525-500 a.C. & Partenza di guerrieri & & Parigi, Louvre F320 & $\begin{array}{c}\text { CVA 8, tav. 73.8-10; Drougou } \\
\text { 1975, 13 A4 }\end{array}$ \\
\hline 12 & FN & Maniera del P. di Antimenes & 525-500 a.C. & $\begin{array}{c}\text { Ritorno di Efesto, Dioniso } \\
\text { assiso fra satiri e menadi }\end{array}$ & & Parigi, Louvre F321 & $\begin{array}{c}\text { CVA 8, tav. 73.4-6; Drougou } \\
\text { 1975, } 14 \text { A9, tav. } 14.1\end{array}$ \\
\hline 13 & FN & P. di Lisyppides & 520 a.C. & Eracle e il Leone Nemeo & & $\begin{array}{c}\text { Francoforte, Goethe-Universität, } \\
\text { Antikensammlung } 144\end{array}$ & Drogou 1975, 12 A1 \\
\hline 14 & FN & $\begin{array}{l}\text { Gruppo di Leagros / P. di } \\
\text { Acheloos? }\end{array}$ & 515 a.C. & Simposio & & Roma, Caltagirone 718 & M\&M-Auction 1980, tav. 29.77 \\
\hline
\end{tabular}


Frankfurter elektronische Rundschau zur Altertumskunde 16 (2011)

\begin{tabular}{|c|c|c|c|c|c|c|c|}
\hline N. & TECNICA & CERAMISTA/PITTORE & DATAZIONE & ICONOGRAFIA & ISCRIZIONI & N. INV. & BIBLIOGRAFIA \\
\hline 15 & FR & Euphronios & 515 a.C. & Morte di Penteo & "Galene, Pentheus" & $\begin{array}{c}\text { Boston (MA), Museum of Fine } \\
\text { Arts } 10.221\end{array}$ & $\begin{array}{c}\text { Drougou } 1975,16 \text {, A22, 60-63, } \\
111 \text { nota } 105 \text {, tav. } 6\end{array}$ \\
\hline 16 & FR & Oltos & 515 a.C. & Atleti & $\begin{array}{l}\text { "Smiky[thos], } \\
\text { Ba[tr]achos, } \\
\text { Dorotheos, } \\
\text { Antiphanes, Epainetos } \\
\text { kalos, Kleainetos, } \\
\text { Alketes, } \\
\text { A[nt]i[m]enes, } \\
\text { haloymenos eisi / } \\
\text { pome, chars [.], " }\end{array}$ & $\begin{array}{c}\text { New York (NY), Metropolitan } \\
\text { Museum 10.210.18 }\end{array}$ & \begin{tabular}{|c|} 
Drougou 1975, 15 A17, tavv. 2 \\
3; Bothmer 1986, fig. 2a-b; \\
Steiner 2007, fig. 10.6 \\
\end{tabular} \\
\hline 17 & FN & Gruppo di Leagros & 510-500 a.C. & Simposio & $\begin{array}{l}\text { iscriz. "nonsense" } \\
\text { escono dalla bocca dei } \\
\text { personaggi }\end{array}$ & $\begin{array}{l}\text { Monaco, Antikensammlungen } \\
\text { SL461 }\end{array}$ & $\begin{array}{c}\text { Drougou 1975, } 14 \text { A15, tav. } \\
27.1\end{array}$ \\
\hline 18 & FN & Gruppo di Leagros & 510-500 a.C. & Simposio & & Tarquinia, Museo Naz. RC6823 & $\begin{array}{c}\text { Drougou 1975, } 14 \text { A12, tav. } \\
5.1-2\end{array}$ \\
\hline 19 & FR & Maniera di Euphronios & 510-500 a.C. & $\begin{array}{c}\text { Giovani in costume tracio } \\
\text { conducono cavalli }\end{array}$ & & Parigi, Louvre G59 & $\begin{array}{l}\text { CVA 8, tavv. 58. 1, 4, 7-59. 1, } \\
\text { 6; Drougou 1975, } 16 \text { A19 }\end{array}$ \\
\hline 20 & FR & Smikros & 510-500 a.C. & $\begin{array}{l}\text { Pescatori? (giovani ed } \\
\text { uomini con reti) }\end{array}$ & & $\begin{array}{c}\text { Malibu (CA), Getty Museum } \\
\text { 83.AE.285 }\end{array}$ & Moon 1983, 148, fig. 10.1a-c \\
\hline 21 & FR & P. di Kleophrades & 510-500 a.C. & Simposio & & $\begin{array}{c}\text { Princeton (NJ), Univ. Museum } \\
1989.69 \\
\end{array}$ & Lissarrague 2001, 29, fig.17 \\
\hline 22 & FR & Smykros & 510-500 a.C. & Cavalieri in costume tracio & $\begin{array}{l}\text { "Delphis, Gnathon, } \\
\text { Philokydes, } \\
\text { Aristaichmos" }\end{array}$ & Roma, Villa Giulia & Godart - De Caro 2007, 86-87 \\
\hline 23 & FR & Smykros & 510-500 a.C. & $\begin{array}{c}\text { Cinque coppie di giovani } \\
\text { (eromenos/erastes) }\end{array}$ & $\begin{array}{l}\text { "Melas, Antias, } \\
\text { Euphronios, Leagros } \\
\text { kalos. Egherthos kalos, } \\
\text { Andriskos, Ambrosios, } \\
\text { Euthydikos" }\end{array}$ & $\begin{array}{l}\text { Malibu (CA), Paul Getty } \\
\text { Museum 82.AE.53 }\end{array}$ & Neer 2002, 100-101, fig. 46 \\
\hline
\end{tabular}


Frankfurter elektronische Rundschau zur Altertumskunde 16 (2011)

\begin{tabular}{|c|c|c|c|c|c|c|c|}
\hline N. & TECNICA & CERAMISTA/PITTORE & DATAZIONE & ICONOGRAFIA & ISCRIZIONI & N. INV. & BIBLIOGRAFIA \\
\hline 24 & FR & Euphronios & 510-500 a.C. & $\begin{array}{c}\text { Quattro etere nude } \\
\text { recumbenti a simposio, una } \\
\text { gioca al kottabos come } \\
\text { "Liebesorakel" }\end{array}$ & $\begin{array}{l}\text { "tin tande latasso, } \\
\text { Leagre; Smikra, } \\
\text { Palaisto, Euphronios } \\
\text { egraphsen, Sekline, } \\
\text { Agape" } \\
\end{array}$ & $\begin{array}{l}\text { San Pietroburgo, Hermitage } \\
\text { ST1670 }\end{array}$ & $\begin{array}{l}\text { Drougou 1975, } 16 \text { A 23, 85-87, } \\
\text { tav. 4.1; Radford 1915, 110-111 }\end{array}$ \\
\hline 25 & FR & Smykros & 510-500 a.C. & Komos & $\begin{array}{l}\text { "Diomnestos, } \\
\text { Mosokles, Antiphanes, } \\
\text { Diodoros, Eukleides" }\end{array}$ & Parigi, Louvre G58 & $\begin{array}{c}\text { Drougou 1975, } 16 \text { A20, 89-90, } \\
\text { tav. } 4.2\end{array}$ \\
\hline 26 & FR & Phintias & 510-500 a.C. & Atleti in palestra & $\begin{array}{l}\text { "Simon, Eoppoki, } \\
\text { Filon, Etearchos; } \\
\text { Ptoiodoros, Sostratos, } \\
\text { Eukrates; Elioas, } \\
\text { Eudemos, Sostratos; } \\
\text { Epilykos, Xenophon, } \\
\text { Phayllos" }\end{array}$ & $\begin{array}{c}\text { Boston (MA), Museum of Fine } \\
\text { Arts } 01.8019\end{array}$ & $\begin{array}{l}\text { Drougou 1975, } 16 \text { A21, tav. 1, } \\
\text { Shapiro 1983, 305-310 }\end{array}$ \\
\hline 27 & FR & Euthymides & 510-500 a.C. & $\begin{array}{c}\text { A, atleti in lotta (Teseo e } \\
\text { Klitos); B, atleti }\end{array}$ & $\begin{array}{l}\text { "Euthymides } \\
\text { egraphsen hopolio, } \\
\text { Theseus, Klytos eyge } \\
\text { naichi, Eythymides } \\
\text { egraphsen ho polio } \\
\text { phylos o[...]ra" } \\
\end{array}$ & Torino, Museo di Antichità 4123 & $\begin{array}{l}\text { Drougou 1975, } 20 \text { B7, tav. 12; } \\
\text { Steiner 2007, fig. 7.21-22 }\end{array}$ \\
\hline 28 & FR & P. di Amasis / P. di Dikaios & 510-500 a.C. & A-B, komos & $\begin{array}{l}\text { "Kydias, Chaire, } \\
\text { Chaire, karta, Dikaios } \\
\text { [--]charchon" }\end{array}$ & Londra, British Museum E767 & $\begin{array}{c}\text { Drougou 1975, } 20 \text { B8, 88-89, } \\
\text { tavv. 10-11; Shapiro 1982, 69- } \\
73\end{array}$ \\
\hline 29 & FR & Oltos & 510-500 a.C. & Opliti su delfini & $\begin{array}{l}\text { "epidelphinos" } \\
\text { (ripetuto sei volte) }\end{array}$ & $\begin{array}{c}\text { New York (NY), Metropolitan } \\
\text { Museum L1979.17.1 }\end{array}$ & Drougou 1975, 15 A18, 67-69 \\
\hline 30 & FN & & 510-490 a.C. & $\begin{array}{l}\text { Cavalieri, alcuni con } \\
\text { cappello tracio; un uomo } \\
\text { con petaso e chitonisco } \\
\text { conduce un cavallo }\end{array}$ & & $\begin{array}{c}\text { New York (NY), Shelby White \& } \\
\text { Leon Levy Collection } 43272\end{array}$ & Bothmer 1991, 148-149, n. 112 \\
\hline 31 & FN (frr.) & & 510 a.C. са & $\begin{array}{l}\text { A, partenza del guerriero; B, } \\
\text { simposio (Eracle con } \\
\text { kantharos, Atena, Dioniso } \\
\text { con kantharos, Hermes) } \\
\end{array}$ & & $\begin{array}{c}\text { Lipsia, Karl Marx Universität } \\
\text { T4217 }\end{array}$ & CVA 2, 28-29, tav. 26.1-4 \\
\hline 32 & FR & Myson & 500-490 a.C. & A, satiri; B, komos & & Atene, Ceramico 2710 & Drougou 1975, 20 B9, tav. 22.1 \\
\hline
\end{tabular}


Frankfurter elektronische Rundschau zur Altertumskunde 16 (2011)

\begin{tabular}{|c|c|c|c|c|c|c|c|}
\hline N. & TECNICA & CERAMISTA/PITTORE & DATAZIONE & ICONOGRAFIA & ISCRIZIONI & N. INV. & BIBLIOGRAFIA \\
\hline 33 & FR & & 500-490 a.C. & Atleti & kalos & Atene, Agora P7240 & $A R V^{2}$ 193.1; Ashmead 1966 \\
\hline 34 & $\mathrm{FN}$ & & 500 a.С. са. & Satiri con le armi di Eracle & & Brussels, Musees Royaux A1312 & $\begin{array}{c}\text { CVA 3, tav. 27. 4a-4b; Drogou } \\
\text { 1975, } 19 \text { B4 }\end{array}$ \\
\hline 35 & FR & P. di Kleophrades & 500 a.C. & Dioniso con satiri e menadi & $\begin{array}{l}\text { Iscriz. "nonsense" alle } \\
\text { spalle o accanto ai } \\
\text { suonatori, vicine alle } \\
\text { bocche dei } \\
\text { protagonisti }\end{array}$ & Parigi, Louvre G57 & $\begin{array}{c}\text { CVA 8, III.I.C.47, III.I.C.48, } \\
\text { tavv. 58.2.5.8, 59.2-3; Drougou } \\
\text { 1975, } 17 \text { A24, tav. } 14.2\end{array}$ \\
\hline 36 & FR & P. di Kleophrades & 500 a.C. & $\begin{array}{c}\text { Eracle e Dioniso fra satiri, in } \\
\text { primo piano rispetto ad una } \\
\text { piattaforma su cui si } \\
\text { dispiegano forme potorie }\end{array}$ & $\begin{array}{l}\text { Iscriz. "nonsense": } \\
\text { "iolele ialiniyne lyn } \\
\text { isyp ylis" }\end{array}$ & Compiegne, Musee Vivenel 1068 & Drougou 1975, 17 A25, tav. 15 \\
\hline 37 & FR (fr.) & Vicino al P. di Kleophrades & 500 a.C. & Atleti & & Atene, Agorà P7240E & Drougou 1975, 17 A26 \\
\hline 38 & FR & Myson & 495 a.C. & Teseo rapisce Antiope & $\begin{array}{l}\text { "Antiopeia, } \\
\text { Androdameia, } \\
\text { Eurypyleia" }\end{array}$ & $\begin{array}{c}\text { Città del Vaticano, Museo } \\
\text { Gregoriano AST428 }\end{array}$ & $\begin{array}{c}\text { Drougou 1975, } 17 \text { A28, 75, tav. } \\
16.1 \\
\end{array}$ \\
\hline 39 & FR & Douris & 490-480 a.C. & Satiri & $\begin{array}{l}\text { "Aristagoras kalos; } \\
\text { Doris egraphsen" }\end{array}$ & Londra, British Museum E768 & $\begin{array}{l}\text { Drougou 1975, 17-18 A30, 65- } \\
\text { 66, tav. 18-19; Robertson 1996, } \\
\text { fig. } 86 \\
\end{array}$ \\
\hline 40 & FR & P. di Syriskos & 480 a.C. & $\begin{array}{l}\text { A, atleti; B, corteggiamento } \\
\text { omoerotico }\end{array}$ & & $\begin{array}{c}\text { Baltimora (MD), Walters Art } \\
\text { Gallery } 48.77\end{array}$ & $\begin{array}{c}\text { Drougou 1975, } 21 \text { B13, tav. } \\
26.1\end{array}$ \\
\hline 41 & FR & P. di Tyszkiewicz & 480 a.C. & Teseo ed il Minotauro & & Roma, Villa Giulia 49796 & $\begin{array}{l}\text { CVA 4, 44-45, tav. 42.1-2; } \\
\text { Drougou 1975, } 18 \text { A35 }\end{array}$ \\
\hline 42 & FR & P. di Pan & 480 a.C. & Ida e Marpessa & & $\begin{array}{l}\text { Monaco, Antikensammlungen } \\
\text { J745 }\end{array}$ & $\begin{array}{l}\text { Drougou 1975, } 18 \text { A33, tav. 17; } \\
\text { Sourvinu Inwood 1975, 109ss. }\end{array}$ \\
\hline 43 & FR & Myson / P. di Eucharides & 480 a.C. & A, oplita; B, komos & & $\begin{array}{c}\text { Berlino, Antikensammlung } \\
1966.14\end{array}$ & $\begin{array}{c}\text { Drougou 1975, } 20 \text { B10, tav. } \\
25.1\end{array}$ \\
\hline 44 & FR & & 475-425 a.C. & Eracle sulla pira, Filottete & & $\begin{array}{c}\text { New York (NY), Malcolm } \\
\text { Weiner } 9949\end{array}$ & $\begin{array}{l}\text { Lissarrague - Thelamon 1983, } \\
152\end{array}$ \\
\hline 45 & FR & Onesimos & 475 a.C. & Centauromachia & & Roma, Villa Giulia 3577 & $\begin{array}{c}\text { CVA 1, III.I.C.4, tavv.19-20, } \\
\text { 3.1-3, 4.1-3; Drougou 1975, } 18 \\
\text { A34, tav. } 16.2\end{array}$ \\
\hline 46 & FR (fr.) & P. di Tyszkiewicz & 470 a.C. са. & Figura femminile & & $\begin{array}{l}\text { Wurzburg, Universität, Martin } \\
\text { von Wagner Museum H5403 }\end{array}$ & CVA 2, 54, fig. 42 , tav. 38.7 \\
\hline
\end{tabular}


Frankfurter elektronische Rundschau zur Altertumskunde 16 (2011)

\begin{tabular}{|c|c|c|c|c|c|c|c|}
\hline N. & TECNICA & CERAMISTA/PITTORE & DATAZIONE & ICONOGRAFIA & ISCRIZIONI & N. INV. & BIBLIOGRAFIA \\
\hline 47 & FN & & 470 a.C. & $\begin{array}{c}\text { A, gigantomachia; B, } \\
\text { cavalieri }\end{array}$ & & $\begin{array}{l}\text { Monaco, Antikensammlungen } \\
\text { L54 }\end{array}$ & Drougou 1975, 19 B5, tav. 28.2 \\
\hline 48 & FR & P. della Yale Oinochoe & 470 a.C. & $\begin{array}{c}\text { A, gara musicale?; B, } \\
\text { giovane in costume tracio } \\
\text { conduce cavalli }\end{array}$ & & $\begin{array}{c}\text { New York (NY), Shelby White \& } \\
\text { Leon Levy Collection } 43262 \\
\end{array}$ & $\begin{array}{c}\text { Bothmer 1991, 172, n.123; } \\
\text { Drougou 1975, } 20 \text { B11 } \\
\end{array}$ \\
\hline 49 & FR & P. della Yale Lekythos & 470 a.C. & $\begin{array}{l}\text { A, Eros in volo con leprotto; } \\
\text { B, giovane con gallo e } \\
\text { cerchio }\end{array}$ & & Berlino, Antikensammlung 3407 & Drougou 1975, 21 B15, tav. 28 \\
\hline 50 & FN & & VI sec. a.C. & Corsa di carri & & $\begin{array}{l}\text { Basilea, Antikenmuseum und } \\
\text { Sammlung Ludwig }\end{array}$ & Drougou 1975, 13 A6; tav. 9.2 \\
\hline 51 & FN & & VI sec. a.C. & $\begin{array}{c}\text { Efesto o Dioniso sul mulo } \\
\text { fra satiri }\end{array}$ & & $\begin{array}{c}\text { New York (NY), Metropolitan } \\
\text { Museum 06.1021.80 }\end{array}$ & Drougou 1975, 14 A11, tav. 8.1 \\
\hline 52 & FN & Vicino al P. di Acheloos & VI sec. a.C. & Komos & Iscriz. "nonsense" & Brussels, Musees Royaux A1652 & $\begin{array}{c}\text { CVA 3, tav. 25.5a-b; Drougou } \\
\text { 1975, 19 B3, tav. 13.1 }\end{array}$ \\
\hline 53 & FN & $\begin{array}{l}\text { Gruppo del P. di Wurzburg } \\
199\end{array}$ & VI sec. a.C. & Dioniso fra satiri e menadi & & Roma, Villa Giulia M445 & Drougou 1975, 13 A5, tav. 9.1 \\
\hline 54 & FN & & V sec. a.C. & Meandro & & $\begin{array}{c}\text { Oxford, Ashmolean Museum } \\
1927.4597\end{array}$ & CVA 1, tav. 48.26 \\
\hline 55 & FN & & V sec. a.C. & A-B komos & & Atene, Museo Nazionale T454 & $\begin{array}{c}\text { Stampolidis - Parlama 2000, } \\
\text { 326-327, n.339 }\end{array}$ \\
\hline 56 & FN & & V sec. a.C. & Komos & & London Market, Bonhams & $\begin{array}{l}\text { Onhams: The Geddes } \\
\text { Collection 2008, n. } 6\end{array}$ \\
\hline 57 & FN & Gruppo E & & Eracle e Gerione & & Francavilla Marittima 4509 & $\begin{array}{c}\text { AMemSocMGr, ns XI-XII } \\
\text { 1970-71, tavv.29a, 30a-b }\end{array}$ \\
\hline 58 & FN & P. Rycroft & & $\begin{array}{c}\text { Dioniso su carro con satiri e } \\
\text { menadi }\end{array}$ & & $\begin{array}{c}\text { Bloomington (IN), Indiana } \\
\text { University Art Museum 75.102.1 }\end{array}$ & $\begin{array}{c}\text { Moon - Berge 1979, 112, 113, } \\
\text { n. } 64\end{array}$ \\
\hline 59 & $\begin{array}{c}\text { FN } \\
\text { (beotico? } \\
\text { ispiraz. } \\
\text { corinzia) }\end{array}$ & & & $\begin{array}{c}\text { Danzatori, combattimento di } \\
\text { galli }\end{array}$ & & Parigi, Rodin TC154 & $\begin{array}{c}\text { CVA 8, tav. 7.7-8; Drougou } \\
\text { 1975, 15 A16a } \\
\end{array}$ \\
\hline 60 & FR (fr.) & & & Atleti & & $\begin{array}{c}\text { Eretria, Archaeological Museum } \\
\text { E5.91.6 }\end{array}$ & Eretria IX, tavv. 21, 25, 88.S37 \\
\hline 61 & FR (fr.) & & & Atleti & & $\begin{array}{c}\text { Malibu (CA), Paul Getty } \\
\text { Museum 95.AE.32 }\end{array}$ & Beazley Archive 28776 \\
\hline
\end{tabular}


Frankfurter elektronische Rundschau zur Altertumskunde 16 (2011)

\begin{tabular}{|c|c|c|c|c|c|c|c|}
\hline N. & TECNICA & CERAMISTA/PITTORE & DATAZIONE & ICONOGRAFIA & ISCRIZIONI & N. INV. & BIBLIOGRAFIA \\
\hline 62 & FN (fr.) & & & Giovani danzanti, animali & & $\begin{array}{l}\text { Malibu (CA), Paul Getty } \\
\text { Museum 82.AE.125 }\end{array}$ & Beazley Archive 28784 \\
\hline 63 & FR (fr.) & & & Figura maschile in corsa & & $\begin{array}{l}\text { Malibu (CA), Paul Getty } \\
\text { Museum 86.AE.225 }\end{array}$ & CVA 7, 24, tav. 348.3 \\
\hline 64 & FR & & & $\begin{array}{l}\text { Figure maschili, alcune con } \\
\text { bastone }\end{array}$ & & Atene, Agorà P16820 & $\begin{array}{l}\text { The Athenian Agora XXX, tav. } \\
64.59\end{array}$ \\
\hline 65 & FR & & & Atleti, pedotriba & & $\begin{array}{l}\text { Malibu (CA), The J. Paul Getty } \\
\text { Museum 78.AE.249A-B }\end{array}$ & Beazley Archive 28867 \\
\hline 66 & FN & & & Komos & & Atene, Agorà P27885 & $\begin{array}{c}\text { The Athenian Agora XXIII, 151, } \\
\text { fig.11, tav. 39.398 }\end{array}$ \\
\hline 67 & $\mathrm{FN}$ & Vicino al P. di Acheloos & & Komos & & Londra, British Museum B299 & Drougou 1975, 14 A14 \\
\hline 68 & $\mathrm{FN}$ & & & A, komos; B, guerriero & & Jena, Universität 340 & Drougou 1975, 15 A16 \\
\hline 69 & FR & & & Atleti & & Atene, Agorà P 7240 & \\
\hline 70 & FR & Maniera di Myson & & Apollo e Artemide & & Heidelberg, Universität & Drougou 1975, 17 A29 \\
\hline 71 & FR & & & A-B komos & & Atene, Ceramico 6264 & Drougou 1975, 21 B12 \\
\hline 72 & FR & Vicino ad Hermonax & & $\begin{array}{l}\text { A, Dioniso e menade; B, } \\
\text { satiro e menade }\end{array}$ & & Rodi, Museo & Drougou 1975, 21 B14 \\
\hline
\end{tabular}

Tav. 2 Attestazioni iconografiche dello psykter nella ceramica attica.

\begin{tabular}{|c|c|c|c|c|c|c|c|}
\hline N. & TECNICA & FORMA & CERAMISTA/PITTORE & DATAZIONE & ICONOGRAFIA & N. INV. & BIBLIOGRAFIA \\
\hline 1 & FN & Oinochoe & Kleisophos / Xenokles & 530-20 a.C. & Komos: lo psykter galleggia all'interno di un cratere a calice & $\begin{array}{c}\text { Atene, Museo } \\
\text { Nazionale } 1045 \\
\end{array}$ & $\begin{array}{c}A B V \text { 432; Drougou 1975, } \\
\text { 38.1; Smith 2010, 322, tav. } \\
\text { 18D, 010, 2.1-3, } \\
\text { III.H.EFGH.3 }\end{array}$ \\
\hline 2 & FN & Coppa & $\begin{array}{l}\text { Maniera del P. di } \\
\text { Andokides }\end{array}$ & 520 a.C. & $\begin{array}{l}\text { Komos: lo psykter galleggia all'interno di un cratere a calice; } \\
\text { da esso attingono due giovani con coppe e mestoli }\end{array}$ & $\begin{array}{l}\text { Essen, Folkwang } \\
\text { Museum A169 }\end{array}$ & $\begin{array}{c}\text { Viersneisel 1990, 261, fig. } \\
\text { 47.a }\end{array}$ \\
\hline
\end{tabular}


Frankfurter elektronische Rundschau zur Altertumskunde 16 (2011)

\begin{tabular}{|c|c|c|c|c|c|c|c|}
\hline $\mathbf{N}$. & TECNICA & FORMA & CERAMISTA/PITTORE & DATAZIONE & ICONOGRAFIA & N. INV. & BIBLIOGRAFIA \\
\hline 3 & FN & Skyphos & & 510 a.C. & $\begin{array}{l}\text { Komos: lo psykter galleggia all'interno di un cratere posto fra } \\
\text { due giovani; quello di destra attinge col mestolo dallo psykter } \\
\text { per riempire un corno }\end{array}$ & Heidelberg 278 & $\begin{array}{l}\text { CVA 1, tav. 42, 7-8; } \\
\text { Drougou 1975, 38.2 }\end{array}$ \\
\hline 4 & FN & Pinax (fr.) & Classe di Atene 581 & $\begin{array}{l}\text { Fine del VI sec. } \\
\text { a.C. }\end{array}$ & $\begin{array}{c}\text { Atena assisa, Ermes, divinità, Menade e Satiro presso uno } \\
\text { psykter }\end{array}$ & Atene, Acropoli 2499 & $\begin{array}{l}A B V \text { 506; Drougou 1975, } \\
38.3 \\
\end{array}$ \\
\hline 5 & FN & $\begin{array}{l}\text { Cratere a } \\
\text { colonnette }\end{array}$ & & $\begin{array}{l}\text { Fine del VI sec. } \\
\text { a.C. }\end{array}$ & & Basilea, collez. priv. & Drougou 1975, 38.4 \\
\hline 6 & FN & Pelike & P. della Vogellpelike & 500-490 a.C. & $\begin{array}{c}\text { Fra un uomo ed un giovane con skyphos e mestolo, galleggia } \\
\text { uno psykter all'interno di un cratere a calice }\end{array}$ & Bonn 574 & $\begin{array}{c}A B V \text { 339.1; Drougou 1975, } \\
38.5\end{array}$ \\
\hline 7 & FN & Coppa & & & & $\begin{array}{l}\text { Venice, California } \\
\text { (ex. Collez. Conte G. } \\
\text { Stroganoff) }\end{array}$ & Drougou 1975, 38.6 \\
\hline 8 & FR & Coppa & Cerchia di Nikosthenes & 520-10 a.C. & $\begin{array}{l}\text { Komos: lo psykter giace a terra fra due giovani, uno dei quali } \\
\text { con skyphos e mestolo }\end{array}$ & $\begin{array}{l}\text { Vienna } \\
\text { Kunsthistorische } \\
\text { Museum } 137\end{array}$ & $\begin{array}{c}A R V^{2} \text { 104.1; Drougou 1975, } \\
38.7\end{array}$ \\
\hline 9 & FR & Coppa & Oltos & 510 a.C. са. & Komos: lo psykter galleggia all'interno di un cratere a calice & $\begin{array}{l}\text { Firenze I B } 20 \text { - } \\
\text { Chicago - Villa Giulia }\end{array}$ & $\begin{array}{c}A R V^{2} \text { 59.58; Drougou 1975, } \\
38.8 \\
\end{array}$ \\
\hline 10 & FR & Coppa & P. di Ambrosios & 510 a.C. са. & Simposio & Villa Giulia & $\begin{array}{c}A R V^{2} \text { 173.5; Drougou 1975, } \\
38.9\end{array}$ \\
\hline 11 & FR & Coppa & P. di Thalia & 510 a.C. са. & $\begin{array}{l}\text { Scene di sesso fra comasti ed etere; una delle figure reca in } \\
\text { mano uno psykter }\end{array}$ & Berlino 3251 & $\begin{array}{l}A R V^{2} \text { 113.7; Par. 332; CVA } \\
\text { 2, tav. 57, 1-2; Drougou } \\
\text { 1975, 38.10 }\end{array}$ \\
\hline 12 & FR & Hydria & & 500 a.C. & Simposio: lo psykter galleggia all'interno di un cratere a calice & $\begin{array}{l}\text { Kassel, Staatliche } \\
\text { Kunstsammlungen } \\
\text { (donaz. priv.) }\end{array}$ & Scheibler 1995, fig. 10 \\
\hline 13 & FR & $\begin{array}{l}\text { Lekythos } \\
\text { (squat) }\end{array}$ & P. di Cerbero / Paseas & 510 a.C. са. & $\begin{array}{l}\text { Lo psykter giace a terra fra un giovane e una fanciulla con } \\
\text { skyphos e mestolo }\end{array}$ & Berlino 1960.32 & $\begin{array}{c}A R V^{2} \text { 1630; Drougou 1975, } \\
38.11\end{array}$ \\
\hline
\end{tabular}


Frankfurter elektronische Rundschau zur Altertumskunde 16 (2011)

\begin{tabular}{|c|c|c|c|c|c|c|c|}
\hline N. & TECNICA & FORMA & CERAMISTA/PITTORE & DATAZIONE & ICONOGRAFIA & N. INV. & BIBLIOGRAFIA \\
\hline 14 & FR & Coppa & & 500 a.C. са. & Komos: giovane in corsa con psykter e bastone & Villa Giulia 14 & $\begin{array}{l}A R V^{2} \text { 1564.5; Drougou } \\
1975,38.12 \\
\end{array}$ \\
\hline 15 & FR & Hydria & P. di Nikoxenos & $\begin{array}{l}\text { Inizi del V sec. } \\
\text { a.C. }\end{array}$ & & Basilea, collez. priv. & Drougou 1975, 38.13 \\
\hline 16 & FR & Oinochoe & & $\begin{array}{l}\text { Inizi del V sec. } \\
\text { a.C. }\end{array}$ & Un giovane trasporta un'hydria e uno psykter & $\begin{array}{l}\text { Copenhagen, Museo } \\
\text { Nazionale Abc } 1056 \\
\end{array}$ & $\begin{array}{l}\text { CVA 3.i, tav. 157.4; } \\
\text { Drougou 1975, 38.14 }\end{array}$ \\
\hline 17 & FR & $\begin{array}{l}\text { Pelike } \\
\text { (frr.) }\end{array}$ & Myson & 490 a.C. са. & Komos: giovane con skyphos e mestolo presso uno psykter & Firenze 249 & $\begin{array}{c}A R V^{2} \text { 238.8; Drougou 1975, } \\
38.15\end{array}$ \\
\hline 18 & FR & Coppa & $\begin{array}{c}\text { Maniera del P. di } \\
\text { Antiphanes / Antiphon }\end{array}$ & 480 a.C. са. & $\begin{array}{l}\text { Komos: giovane con skyphos e mestolo; alle sue spalle, uno } \\
\text { psykter galleggia nel cratere a calice. }\end{array}$ & Compiègne 1102 & $\begin{array}{c}A R V^{2} \text { 341.1; Drougou 1975, } \\
\text { 38.16 }\end{array}$ \\
\hline 19 & FR & $\begin{array}{c}\text { Coppa } \\
\text { (frr.) }\end{array}$ & $\begin{array}{c}\text { Maniera del P. di } \\
\text { Antiphanes /Antiphon }\end{array}$ & 480 a.C. са. & Figura maschile con mestolo presso uno psykter & Greifswald 316 & $\begin{array}{c}A R V^{2} \text { 342.2; Drougou 1975, } \\
38.17 \\
\end{array}$ \\
\hline 20 & FR & Coppa & Makron & 480 a.C. са. & $\begin{array}{l}\text { Un'etera con mestolo e coppa si appresta ad attingere da uno } \\
\text { psykter che galleggia in un cratere a calice }\end{array}$ & $\begin{array}{l}\text { Berkeley, California } \\
8.2184 \\
\end{array}$ & $\begin{array}{c}A R V^{2} \text { 475.259; CVA 1, 40, } \\
\text { tav. } 34.1 \text { a-c; Drougou 1975, } \\
38.18\end{array}$ \\
\hline 21 & FR & Lekythos & P. di Pan & 480 a.C. са. & $\begin{array}{l}\text { Lo psykter giace al terra, fra una donna seduta con skyphos e } \\
\text { un'ancella con patera e mestolo }\end{array}$ & $\begin{array}{l}\text { Haverford College, } \\
\text { Comfort } 24\end{array}$ & $\begin{array}{c}A R V^{2} \text { 557.116; Drougou } \\
\text { 1975, 38.19 } \\
\end{array}$ \\
\hline 22 & FR & Coppa & Epiktetos & & Simposio: giovane su kline, con coppa e psykter & $\begin{array}{l}\text { New York (NY) } \\
\text { Market, Christie's }\end{array}$ & $\begin{array}{c}\text { Christie, Manson \& Woods, } \\
\text { sale catalogue: New York } \\
\text { 9.12.2010, 69, n.104 }\end{array}$ \\
\hline 23 & FR & & & & Komos: figura maschile (?) presso uno psykter & $\begin{array}{c}\text { Firenze, Museo } \\
\text { Archeologico Etrusco } \\
\text { 19B13 } \\
\end{array}$ & CVA 1, III.I.22, tav. 19.13 \\
\hline
\end{tabular}


Frankfurter elektronische Rundschau zur Altertumskunde 16 (2011)

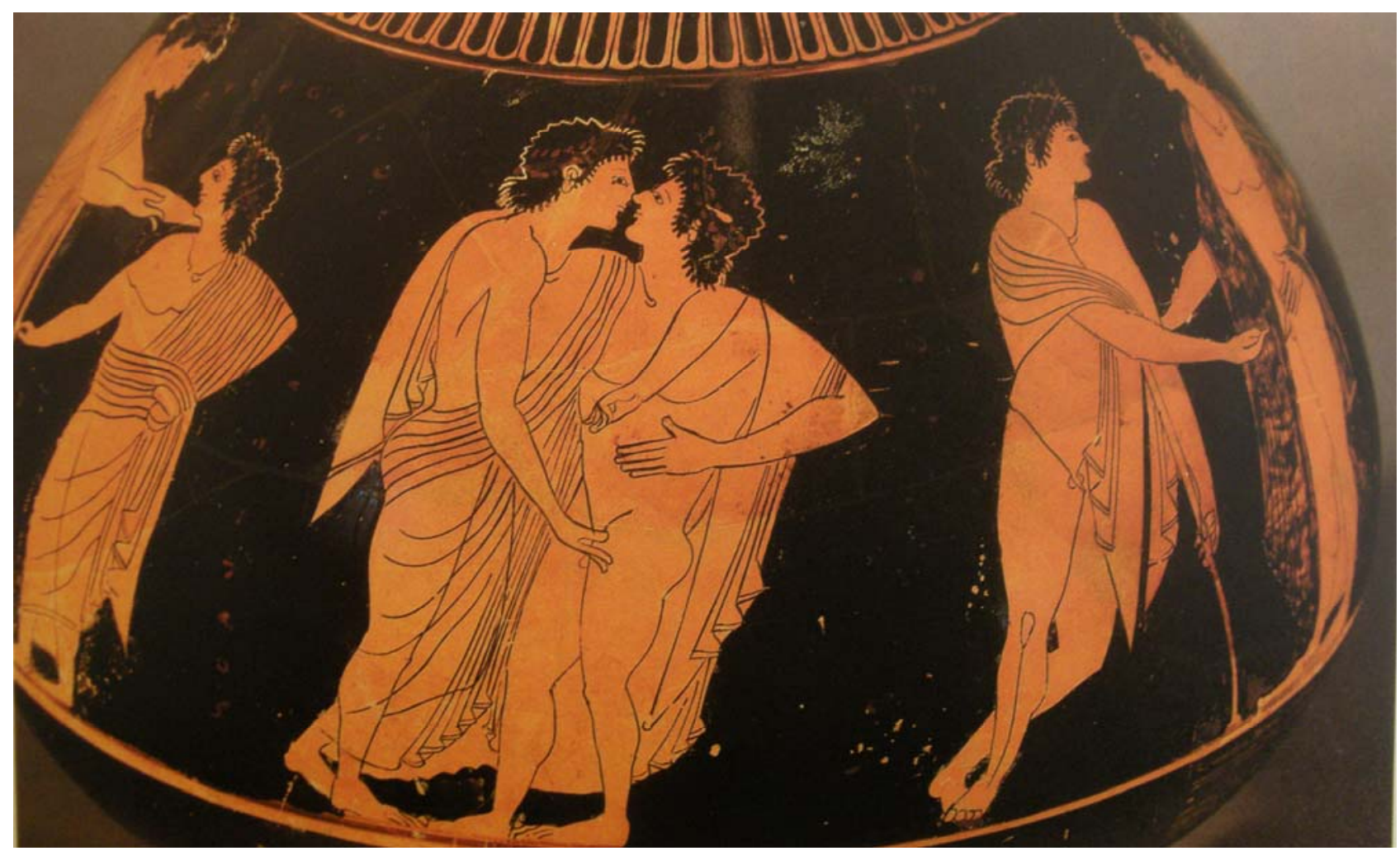

Fig. 1 Psykter Malibu 82.AE.53, tav. 1, n. 23 (GIULIANI ET ALII 1991, n. 60). 
Frankfurter elektronische Rundschau zur Altertumskunde 16 (2011)

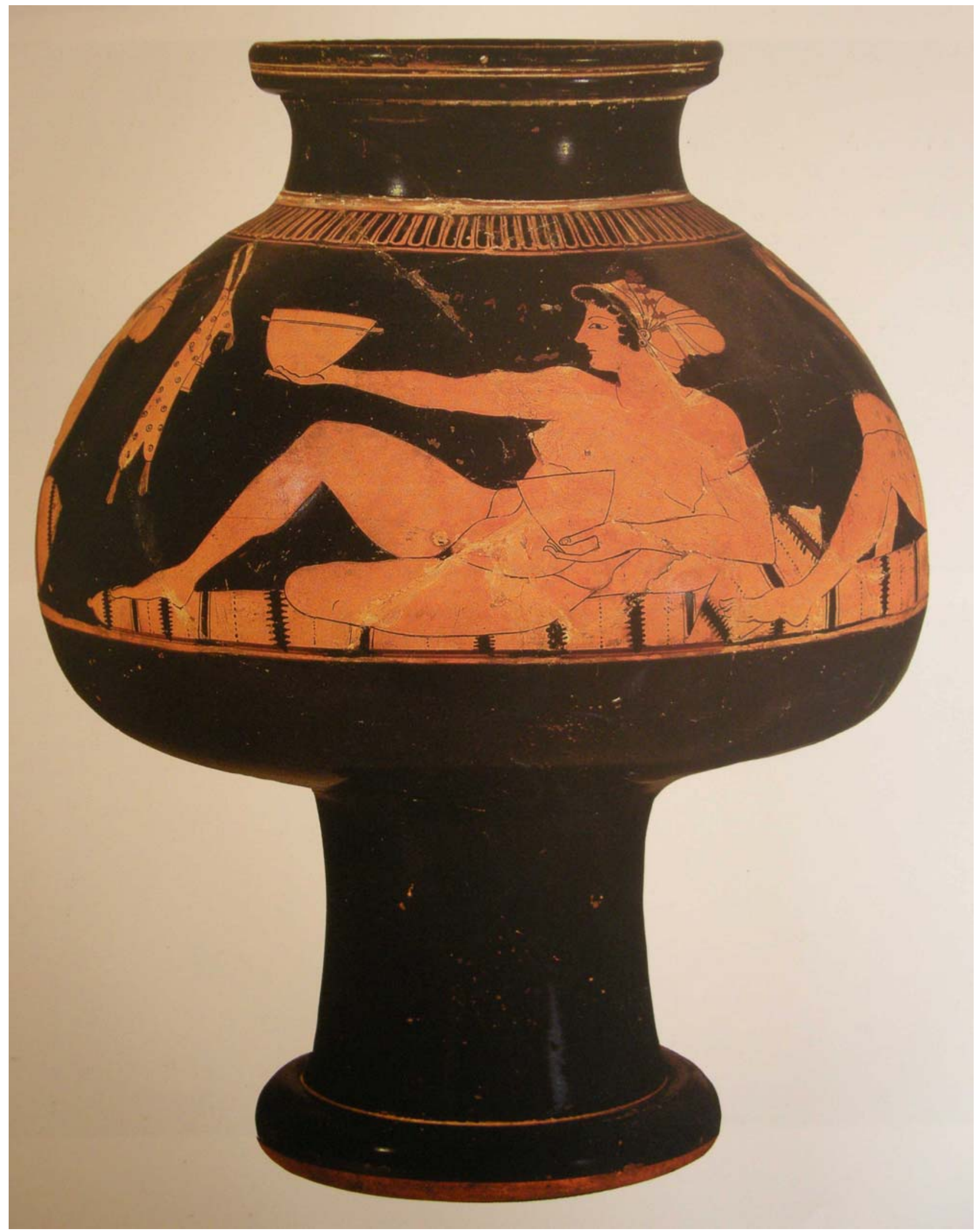

Fig. 2 Psykter San Pietroburgo, Hermitage ST1670, tav. 1, n. 24 (GIULIANI ET ALII 1991, n. 33). 
Frankfurter elektronische Rundschau zur Altertumskunde 16 (2011)

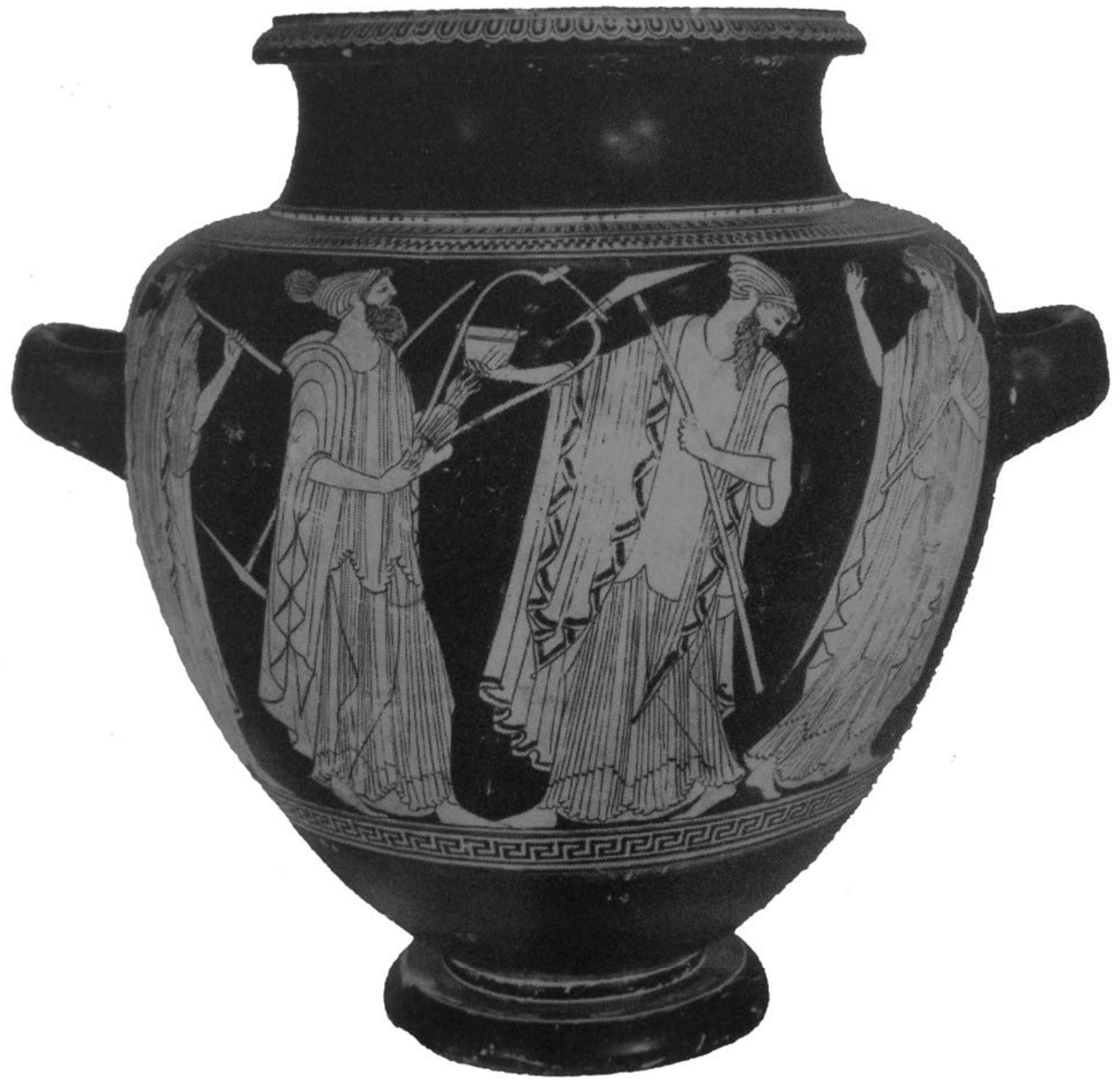

Fig. 3 Stamnos Madrid 11009, Komos “anacreontico. I quarto del V sec. a.C. (NEER 2002, fig. 2). 


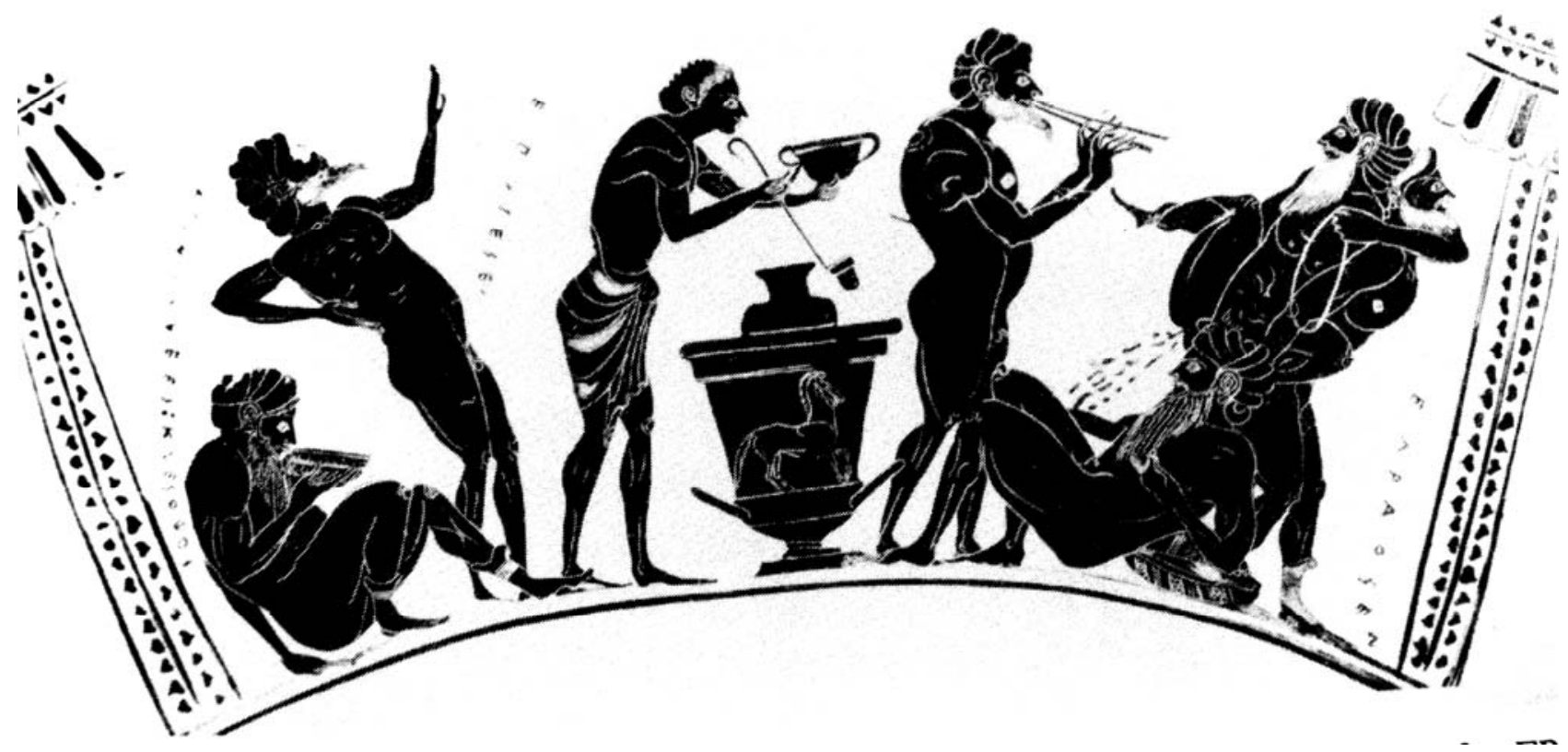

Fig. 4 Oinochoe Atene, Museo Naz. 1045, tav. 2 n. 1 (NEER 2002, fig. 4).

\section{Indirizzo dell'autore:}

Dott.ssa Catia Trombetti

Assegnista di ricerca in Archeologia Classica

Università degli Studi di Perugia

Facoltà di Lettere e Filosofia

Dipartimento di Scienze Storiche dell'Antichità

e-mail: catiatrombetti@yahoo.it 